Article

\title{
Transcriptomic Characterizations of the Molecular Mechanisms Regulating Lipid Production and Composition in Mortierella alpina in Response to Cold Stress
}

\author{
Jiali Ren ${ }^{1,2,+}$, Haijun Wang ${ }^{2,3,+}$, Fengjie Sun ${ }^{4} \mathbb{D}$, Chunxiao Meng ${ }^{1,5}$, Xiangyu Zhu ${ }^{1,2}$, Zuoxi Wei ${ }^{1,2}$, \\ Zhengquan Gao ${ }^{1,5, *}$ and Demao $\mathrm{Li}^{2,6, *}$
}

check for updates

Citation: Ren, J.; Wang, H.; Sun, F.; Meng, C.; Zhu, X.; Wei, Z.; Gao, Z.; Li, D. Transcriptomic Characterizations of the Molecular Mechanisms Regulating Lipid Production and Composition in Mortierella alpina in Response to Cold Stress. Agronomy 2022, 12, 599. https://doi.org/ 10.3390/agronomy12030599

Academic Editor: Caterina Morcia

Received: 5 February 2022

Accepted: 26 February 2022

Published: 28 February 2022

Publisher's Note: MDPI stays neutral with regard to jurisdictional claims in published maps and institutional affiliations.

Copyright: (C) 2022 by the authors. Licensee MDPI, Basel, Switzerland. This article is an open access article distributed under the terms and conditions of the Creative Commons Attribution (CC BY) license (https:// creativecommons.org/licenses/by/ $4.0 /)$.
1 School of Life Sciences, Shandong University of Technology, Zibo 255049, China; renjl@tib.cas.cn (J.R.); mengchunxiao@sdut.edu.cn (C.M.); zhuxy@tib.cas.cn (X.Z.); 20410010840@stumail.sdut.edu.cn (Z.W.)

2 Tianjin Key Laboratory for Industrial Biological Systems and Bioprocessing Engineering, Tianjin Institute of Industrial Biotechnology, Chinese Academy of Sciences, Tianjin 300308, China; wanghj@tib.cas.cn

3 College of Biotechnology, Tianjin University of Science and Technology, Tianjin 300222, China

4 School of Science and Technology, Georgia Gwinnett College, Lawrenceville, GA 30043, USA; fsun@ggc.edu

School of Pharmacy, Binzhou Medical University, Yantai 264003, China

6 National Innovation Centre for Synthetic Biology, Tianjin 300308, China

* Correspondence: gaozhengquan@sdut.edu.cn (Z.G.); li_dm@tib.cas.cn (D.L.); Tel.: +86-187-6696-1859 (Z.G.); +86-022-8486-1932 (D.L.)

$+\quad$ These authors contributed equally to this work.

\begin{abstract}
Cold stress causes changes in the lipid composition of Mortierella alpina. In order to investigate the molecular mechanisms of $M$. alpina in response to cold stress, transcriptome analyses were performed on three groups of M. alpina cultured at (1) $25^{\circ} \mathrm{C}$, (2) $15^{\circ} \mathrm{C}$, and (3) first at $25{ }^{\circ} \mathrm{C}$ for 2 days and then $15^{\circ} \mathrm{C}$ for 7 days, respectively. The results of a gas chromatography-mass spectrometry (GC-MS) analysis suggested that, compared with $25^{\circ} \mathrm{C}$ conditions, dry weight and lipid production were significantly decreased in $\mathrm{M}$. alpina grown at $15^{\circ} \mathrm{C}$, with a total of 1552 differentially expressed genes (DEGs) identified in response to cold stress. The quantitative real-time PCR (qRT-PCR) analysis was conducted to verify the expression patterns of six DEGs involved in lipid metabolism. Results of the enrichment analyses of the DEGs based on the Gene Ontology (GO) and the Kyoto Encyclopedia of Genes and Genomes (KEGG) databases showed that fatty acid compositions were regulated by genes involved in the glycolysis pathway, fatty acid synthesis, the pentose phosphate pathway, the glycerolipid pathway, the tricarboxylic acid (TCA) cycle, and the glycerophospholipid pathway. Our study provided solid experimental evidence and novel insights into the metabolic engineering and the molecular mechanisms regulating the response to cold stress in M. alpina.
\end{abstract}

Keywords: Mortierella alpina; cold stress; transcriptome; unsaturated fatty acids; glycolysis; tricarboxylic acid cycle; fatty acid synthesis; pentose phosphate pathway; glycerolipid pathway; glycerophospholipid pathway

\section{Introduction}

Microbial food and microbial energy are important components of the microbial industry in agriculture, helping effectively alleviate severe societal problems, such as increasing food demand, energy shortages, and environmental pollution worldwide. As an oleaginous fungal species of the class Zygomycetes, Mortierella alpina has shown a wide range of applications in the fields of microbial food and microbial energy. This fungus is capable of accumulating high contents of lipids in its mycelia. These lipids are rich in arachidonic acid (ARA), which is a type of n-6 polyunsaturated fatty acid (PUFA) [1]. As an essential type of fatty acid for human nutrition, ARA is found in high concentrations in several vital tissues, such as brain, retina, and testis, in humans [2]. ARA also plays an important role in the growth and development of infants. In particular, the application of 
ARA in infant formulas is necessary to achieve the optimal development of membrane-rich systems, such as brain and retina [3,4]. Although ARA is generally extracted from animal livers, tuna, and egg yolk, ARA contents in these resources are generally low [5]. With promising prospects, $M$. alpina has been identified as a commercial source of ARA for its incorporation into infant formula [6].

In recent years, the establishment of advanced technologies, such as high-throughput sequencing and gas chromatography-mass spectrometry (GC-MS), has provided novel strategies for studies of microbiomes and synthetic biology to further explore the functions of genes in agricultural microbes. Furthermore, the high-transformation strains have been screened to promote agricultural microbial industry. For example, $M$. alpina uses the classic "fatty acid synthase pathway" rather than the less common "polyketide synthase pathway" (e.g., found in Schizochytrium limacinum) to synthesize PUFAs. With various types of mutants available, this fungal species has been established as a model organism for molecular analyses [7]. Furthermore, the genome of $M$. alpina has been sequenced, with its fatty acid metabolic pathway mapped and its main types of lipids determined, significantly facilitating investigations of the molecular mechanisms underlying the production and metabolism of lipids in this fungal species [7,8]. For example, gene expression of M. alpina under nitrogen stress has been investigated by transcriptomic analysis, demonstrating that the pentose phosphate pathway plays an important role in lipid production in M. alpina [9]. Furthermore, proteomic analysis has revealed a total of 171 differentially expressed proteins (DEPs) showing significant alteration in expression during the aging process in $M$. alpina [10]. Moreover, multi-omics analysis has quantified the global resource allocation by M. alpina in response to nutrient stress [11].

It is well-known that under cold stress the lipid compositions of $M$. alpina show a large number of variations, with some alterations presenting significant commercial value. However, transcriptome analyses exploring the molecular mechanisms underlying lipid biosynthesis and accumulation in $M$. alpina under cold stress are still sparse. For example, studies have shown that, at room temperature, $M$. alpina hardly accumulates any amount of $\omega-3$ eicosatetraenoic acid (ETA). However, an increased amount of ETA (accounting for $42 \%$ of the total lipids) has been identified in M. alpina S14 transformants under cold stress, with the endogenous gene encoding $\omega-3$ desaturase transformed [12]. Under low temperature conditions, M. alpina also produces a type of n-3 PUFA, i.e., eicosapentaenoic acid (EPA). As a type of essential fatty acid, EPA is important for maintaining fundamental biological functions in humans [13]. Studies have shown that EPA possesses many health-promoting properties, such as the prevention and treatment of cardiovascular diseases, Alzheimer's disease, inflammatory disease, cancer, and schizophrenia, making the demand for EPA rapidly increase in the fields of pharmacy, medicine, and nutrition [14]. Deep-sea fish oil is currently the principal commercial source of EPA [15]. However, it is believed that globally, deep-sea fish oil has already reached its maximum level of production [16]. Furthermore, this source of EPA has shown several disadvantages in its commercial applications, such as its high cholesterol content, poor taste, and heavy metal contamination [17].

To date, agricultural microorganisms have significantly enhanced scientific and technological advancements to accelerate development in agriculture. As a high oil-producing microorganism, M. alpina has shown a wide range of applications in microbial energy, microbial feed, and microbial food. In particular, various fermentation strategies, including temperature shift [18], pH shift [19,20], oxygen supply $[18,21]$, consumed carbon to nitrogen $(\mathrm{C} / \mathrm{N})$ ratio [22,23], and precursor supply [22], have been developed and performed to regulate the fatty acid profile and, in particular, enhance lipid accumulation in M. alpina. Although many of these fermentation techniques are effective in changing the lipid contents and the fatty acid profile, the molecular mechanisms regulating the related biological response in $M$. alpina to the environmental changes remain unclear. Furthermore, the metabolic pathway of lipid synthesis in M. alpina has been well established, with several genes involved in the synthesis of lipids identified and transformed into different hosts to increase production of lipids $[7,10]$. The goals of our study were to investigate 
the molecular response in M. alpina cultured at three different temperatures (i.e., $25^{\circ} \mathrm{C}$, $15^{\circ} \mathrm{C}$, and first $25^{\circ} \mathrm{C}$ and then $15^{\circ} \mathrm{C}$ ) based on high-throughput RNA-Seq analysis and to identify the metabolic pathways and related genes regulating the production of lipids in $M$. alpina. The transcriptome of $M$. alpina was constructed based on the de novo assembly of RNA-Seq reads. The results showed that, under cold stress, both the dry weight of fungal spores and lipid production were significantly decreased in M. alpina. A total of 1552 differentially expressed genes (DEGs) of M. alpina were identified in response to cold stress. Furthermore, the DEGs involved in the glycolysis pathway, fatty acid synthesis, the pentose phosphate pathway, the glycerolipid pathway, the tricarboxylic acid (TCA) cycle, and the glycerophospholipid pathway regulated the changes in the production of acetyl-CoA and NADPH, ultimately affecting the production and composition of lipids in M. alpina.

\section{Materials and Methods}

\subsection{Microorganisms and Culture Conditions}

The fungal strain M. alpina ATCC 32222 (CapitalBiol Technology Co., Ltd., Beijing, China) was inoculated on a potato dextrose agar (PDA) plate and cultured at $25^{\circ} \mathrm{C}$. A total of $5 \mathrm{~mL}$ sterile distilled water was added to the PDA plate to collect the fungal spores. The suspension of spores was adjusted to a density of approximately $10^{7}$ spores $/ \mathrm{mL}$ and then inoculated $(5 \%, v / v)$ into the spore culture medium (containing $50.0 \mathrm{~g} / \mathrm{L}$ glucose, $15.0 \mathrm{~g} / \mathrm{L}$ soybean meal, $3.0 \mathrm{~g} / \mathrm{L} \mathrm{KH}_{2} \mathrm{PO}_{4}$, and $\left.1.0 \mathrm{~g} / \mathrm{L} \mathrm{Na}_{2} \mathrm{SO}_{4}\right)$ in a $250 \mathrm{~mL}$ flask, shaken at $200 \mathrm{rpm}$. The spores were cultured for $3.5 \mathrm{~d}$ at $25^{\circ} \mathrm{C}$ and then inoculated $(10 \%, v / v)$ into the fermentation medium (containing $50.0 \mathrm{~g} / \mathrm{L}$ glucose, $30.0 \mathrm{~g} / \mathrm{L}$ soybean meal, $3.0 \mathrm{~g} / \mathrm{L}$ $\mathrm{KH}_{2} \mathrm{PO}_{4}, 1.0 \mathrm{~g} / \mathrm{L} \mathrm{Na}_{2} \mathrm{SO}_{4}, 0.5 \mathrm{~g} / \mathrm{L} \mathrm{CaCl}_{2} \cdot 2 \mathrm{H}_{2} \mathrm{O}$, and $0.5 \mathrm{~g} / \mathrm{L} \mathrm{MgCl} \cdot 6 \mathrm{H}_{2} \mathrm{O}$ ) in a $250 \mathrm{~mL}$ flask, shaken at $200 \mathrm{rpm}$. Based on the results of our preliminary experiments, three experimental groups were set up: (1) the TA group was cultured at $25^{\circ} \mathrm{C}$ and fermentation was stopped after $7 \mathrm{~d}$; (2) in the TB group, the temperature was set at $15^{\circ} \mathrm{C}$ and fermentation was terminated after $7 \mathrm{~d}$; (3) in the TC group, the temperature was first set at $25^{\circ} \mathrm{C}$ for $5 \mathrm{~d}$ and then $15^{\circ} \mathrm{C}$ for $2 \mathrm{~d}$ prior to the termination of fermentation.

\subsection{Lipid Analysis}

The total lipids were extracted by the acid-heating procedure. Specifically, the fungal mycelia were freeze-dried in a vacuum and then ground to mix with $50 \% \mathrm{HCl}$, after which they were incubated in a water bath $\left(75^{\circ} \mathrm{C}\right)$ for $2 \mathrm{~h}$. Then, the total lipids were extracted using n-hexane 3-5 times, with the n-hexane removed under nitrogen. In order to analyze the fatty acid composition, the total lipids were converted into fatty acid methyl esters (FAMEs) by saponification and esterification based on our previous studies [24]. The FAMEs were analyzed by GC-MS (GC2010, Shimadzu, Kyoto, Japan) using a flame ionization detector (FID) and a SP-2560 capillary column $(100 \mathrm{~m} \times 0.25 \mathrm{~mm} \times 0.20 \mu \mathrm{m})$. Supelco ${ }^{\circledR} 37$ Component FAME Mix (Sigma-Aldrich, Beijing, China) was used as a standard to identify the FAMEs in the GC analysis.

\subsection{RNA Extraction, Library Construction, and Illumina Sequencing}

The procedures of sample collection and RNA extraction were the same as those reported in our previous study [24]. The RNA-Seq analysis was performed on one biological replicate in each group of samples. The accuracy of the RNA-Seq data and the expression patterns of genes identified based on the RNA-Seq analysis were further verified by quantitative real-time PCR (qRT-PCR) analysis. RNA integrity was determined in an Agilent 2100 Bioanalyzer using the Agilent Eukaryote Total RNA Nano kit (Agilent, Shanghai, China). The pair-end index libraries were constructed using the NEBNext ${ }^{\circledR}$ UltraTM Directional RNA Library Prep Kit for Illumina ${ }^{\circledR}$ according to the manufacturer's protocol (New England BioLabs, Inc., Shanghai, China). These libraries (Table S1) were sequenced using the Illumina HiSeq2500 System (Jiangsu Jinweizhi, Tianjin, China). 


\subsection{RNA-Seq Analysis, De Novo Assembly, and Functional Annotation}

The raw reads of RNA-Seq were filtered using Trimmomatic (version 0.30) to remove the adaptors and ambiguous nucleotides (i.e., the Ns at the end of the reads) and to eliminate sequences shorter than $75 \mathrm{bp}$ to obtain the clean reads. The clean reads of three samples (i.e., the TA, TB, and TC groups) were assembled using Trinity (version r2013-0225 ) and then further clustered into unigenes using the TIGR Gene Indices clustering tools (TGICL) [25]. The clean data were mapped onto the unigenes using bowtie2 (version 2.1.0) software to compare short reads to quantify the unigenes. The unigenes were annotated using the Basic Local Alignment Search Tool (BLAST) analysis $\left(E\right.$ value $<10^{-5}$ ) based on the non-redundant $(\mathrm{Nr})$ protein database of the National Center for Biotechnology Information (NCBI; https:/ / www.ncbi.nlm.nih.gov/; accessed on 12 March 2020). The functions of the unigenes were annotated by Gene ontology (GO) analysis using the Blast2GO platform [26]. The proteins encoded by the unigenes were classified based on the Cluster of Orthologous Groups (COG) database [27]. The metabolic pathways were enriched by the unigenes based on the Kyoto Encyclopedia of Genes and Genomes (KEGG) database [28].

\subsection{Analysis of Differentially Expressed Unigenes}

The gene expression levels were estimated by RSEM (version 1.2.4) using the fragments per kilobase of transcript per million mapped reads (FPKM) method [29] to obtain the FPKM values of unigenes in each sample by mapping clean reads of RNA-Seq back onto unigenes. Differential expression analysis was conducted using the DESeq R package [30]. The DEGs were identified based on a $p$-value $<0.05$ and fold changes $>2$ or $<-2$ with the $p$-value adjusted using the Benjamini-Hochberg procedure for controlling the false discovery rate (FDR).

\subsection{Quantitative Real-Time PCR Analysis}

A FastKing RT Kit with gDNAase (Tiangen, Beijing, China) was applied to synthesize the cDNAs, following the manufacturer's instructions. A qRT-PCR analysis was performed on a Bio-Rad CFX96 Real-Time PCR System using the PowerUp ${ }^{\text {TM }}$ SYBR ${ }^{\text {TM }}$ Green Master Mix (Thermo Fisher Scientific, Shanghai, China), based on the manufacturer's instructions. All primers used in this study are listed in Table S2.

\section{Results}

\subsection{Effects of Temperature on Fatty Acid Compositions in Mortierella alpina}

Temperature has been shown to have a significant effect on lipid production in M. alpina. In order to investigate the changes in fatty acid composition in M. alpina under cold stress, we analyzed the variations in the contents of lipids produced by M. alpina cultured at three different temperatures (Table 1). Compared with the TA group of $M$. alpina, the lipid contents and biomass in the $\mathrm{TB}$ group were significantly decreased to $9.89 \%$ and $32.54 \%$, respectively. Similarly, the lipid contents and biomass were significantly decreased in the TC group in comparison to the TA group.

Table 1. Lipid contents and biomass in three groups (i.e., TA, TB, and TC) of Mortierella alpina under three different temperatures. The condition " $25^{\circ} \mathrm{C}-15^{\circ} \mathrm{C}$ " indicates the temperature first at $25^{\circ} \mathrm{C}$ for $5 \mathrm{~d}$ and then at $15^{\circ} \mathrm{C}$ for $2 \mathrm{~d}$. The symbol “**” indicates a significant difference set at a $p$-value of 0.01 in comparison to the TA group.

\begin{tabular}{ccc}
\hline Sample & Lipid Content (\%) & Biomass (g/L) \\
\hline $\mathrm{TA}\left(25^{\circ} \mathrm{C}\right)$ & $17.59 \pm 0.27$ & $38.04 \pm 0.24$ \\
$\mathrm{~TB}\left(15^{\circ} \mathrm{C}\right)$ & $9.89 \pm 0.41^{* *}$ & $32.54 \pm 0.52^{* *}$ \\
$\mathrm{TC}\left(25^{\circ} \mathrm{C}-15^{\circ} \mathrm{C}\right)$ & $15.58 \pm 0.13^{* *}$ & $36.03 \pm 0.34^{* *}$ \\
\hline
\end{tabular}

The lipids in M. alpina were then methylated and detected by GC-MS. We measured the contents of four types of saturated fatty acids, i.e., tetradecanoic acid, palmitic acid, 
stearic acid, and arachidic acid, as well as eight types of unsaturated fatty acids, i.e., oleic acid, linoleic acid, r-linolenic acid, eicosenoic acid, eicosadienoic acid, eicosatrienoic acid, ARA, and EPA (Table 2). Under the normal temperature conditions, the proportion of unsaturated fatty acids was higher than that of saturated fatty acids, i.e., $78.36 \%$ and $19.87 \%$, respectively. Under the cold stress condition, the concentration of saturated fatty acids was increased to $26.08 \%$, while the concentration of unsaturated fatty acids was reduced to $72.38 \%$. Similar reactions were observed when $M$. alpina was cultivated first at $25{ }^{\circ} \mathrm{C}$ for 2 days and then transferred to cold stress. The proportion of saturated fatty acids was relatively higher than that of saturated fatty acids, i.e., $20.49 \%$ and $78.22 \%$, respectively. Both types of cold stress application induced three types of saturated fatty acids, i.e., tetradecanoic acid, palmitic acid, and stearic acid, and reduced one type, i.e., arachidic acid. Under cold stress, three types of unsaturated fatty acids, oleic acid, linoleic acid, and r-linolenic acid, were reduced, while two other types, eicosatrienoic acid and EPA, were increased. Another three types of unsaturated fatty acids, eicosenoic acid, eicosadienoic acid, and ARA, showed different reactions under 7-day cold stress and 2-day cold stress, i.e., a reduction in TB and an increase in TC in comparison to TA.

Table 2. Effects of temperature on the fatty acid composition (\%) in the total lipids of three groups (i.e., TA, TB, and TC) of Mortierella alpina under three different temperatures. The condition " $25{ }^{\circ} \mathrm{C}-15{ }^{\circ} \mathrm{C}$ " indicates the temperature first at $25^{\circ} \mathrm{C}$ and then at $15^{\circ} \mathrm{C}$. The symbols "** and "*** indicate significant differences set at $p$-values of 0.05 and 0.01 , respectively, in comparison to the TA group.

\begin{tabular}{cccc}
\hline Fatty Acid & TA $\left(\mathbf{2 5}{ }^{\circ} \mathbf{C}\right)$ & TB $\left(\mathbf{1 5}{ }^{\circ} \mathbf{C}\right)$ & TC $\left(\mathbf{2 5}{ }^{\circ} \mathbf{C}-\mathbf{1 5}{ }^{\circ} \mathbf{C}\right)$ \\
\hline Tetradecanoic acid (C14:0) & $0.29 \pm 0.035$ & $0.678 \pm 0.061^{* *}$ & $0.35 \pm 0.027^{*}$ \\
Palmitic acid (C16:0) & $7.84 \pm 0.145$ & $9.60 \pm 0.225^{* *}$ & $8.33 \pm 0.232^{* *}$ \\
Stearic acid (C18:0) & $11.20 \pm 0.917$ & $15.30 \pm 0.874^{* *}$ & $11.29 \pm 0.657$ \\
Oleic acid (C18:1n9t) & $0.06 \pm 0.013$ & $0.07 \pm 0.011^{*}$ & $0.05 \pm 0.013^{*}$ \\
Oleic acid (C18:1n9c) & $7.58 \pm 0.750$ & $5.36 \pm 0.53^{* *}$ & $7.36 \pm 0.032^{* *}$ \\
Linoleic acid (C18:2n6c) & $5.82 \pm 0.232$ & $4.11 \pm 0.211^{* *}$ & $5.44 \pm 0.307^{* *}$ \\
r-linolenic acid (C18:3n6) & $4.34 \pm 0.371$ & $3.61 \pm 0.341^{* *}$ & $4.12 \pm 0.429^{* *}$ \\
Arachidic acid (C20:0) & $0.58 \pm 0.043$ & $0.55 \pm 0.026^{*}$ & $0.56 \pm 0.033^{* *}$ \\
Eicosenoic acid (C20:1) & $0.30 \pm 0.029$ & $0.17 \pm 0.014^{* *}$ & $0.32 \pm 0.028^{*}$ \\
Eicosadienoic acid (C20:2) & $0.48 \pm 0.054$ & $0.41 \pm 0.043^{* *}$ & $0.52 \pm 0.056^{* *}$ \\
Eicosatrienoic acid (C20:3) & $4.40 \pm 0.238$ & $7.45 \pm 0.657^{* *}$ & $4.66 \pm 0.382^{* *}$ \\
Arachidonic acid (C20:4) & $56.40 \pm 1.835$ & $51.77 \pm 1.677^{* *}$ & $56.59 \pm 1.971 *$ \\
Eicosapentaenoic acid (C20:5) & $0.01 \pm 0.001$ & $0.22 \pm 0.014^{* *}$ & $0.22 \pm 0.021^{* *}$ \\
\hline
\end{tabular}

\subsection{RNA-Seq and Transcriptome Analyses of Lipid Synthesis in Mortierella alpina under Cold Stress}

The three groups of samples of M. alpina were sequenced by Hiseq2500 technology to obtain the raw data of RNA-Seq, which were further processed by Trimmomatic to remove the adapters and low-quality sequences to obtain the clean data for subsequent analyses (Table 3). The transcriptome of $M$. alpina was constructed based on the de novo assembly of clean reads using Trinity, and the sequence clustering software TGICL was used to obtain the non-redundant unigene sequences (Table 4). Among the total of 22,059 unigenes, the shortest and the longest unigenes were $201 \mathrm{bp}$ and 15,549 bp, respectively, with an average length of $1429.89 \mathrm{bp}$ and a GC content of $53.23 \%$. The statistics for the clean data mapped onto the unigenes based on bowtie2 are given in Table 5 . 
Table 3. Statistics of RNA-Seq data for three groups (i.e., TA, TB, and TC) of Mortierella alpina. The statistics for the clean data based on Trimmomatic are given in parentheses.

\begin{tabular}{cccccccc}
\hline Sample & Length (nt) & Reads & Nucleotide & Q20 (\%) & Q30 (\%) & GC (\%) & N (ppm) \\
\hline \multirow{2}{*}{ TA } & 101 & $55,701,908$ & $5,625,892,708$ & 95.57 & 90.44 & 53.72 & 933.86 \\
& $(100.27)$ & $(46,514,296)$ & $(4,663,901,130)$ & $(99.69)$ & $(96.78)$ & $(53.51)$ & $(0.46)$ \\
TB & 101 & $48,534,748$ & $4,902,009,548$ & 95.45 & 90.06 & 53.88 & 927.96 \\
& $(100.24)$ & $(40,287,982)$ & $(4,038,356,115)$ & $(99.67)$ & $(96.62)$ & $(53.61)$ & $(0.46)$ \\
TC & 101 & $65,639,098$ & $6,629,548,898$ & 95.89 & 90.77 & 54.25 & 938.59 \\
& $(100.24)$ & $(55,154,048)$ & $(5,528,511,690)$ & $(99.69)$ & $(96.73)$ & $(54.00)$ & $(0.46)$ \\
\hline
\end{tabular}

Table 4. Statistics (\%) for the de novo assembly of the transcriptome in Mortierella alpina based on Trinity and the TIGR Gene Indices clustering tools (TGICL).

\begin{tabular}{|c|c|c|c|c|c|c|c|}
\hline Assembled Unit & Total & $<200 \mathrm{nt}$ & $(200-500) n t$ & $(500-1000) \mathrm{nt}$ & $(1000-1500) \mathrm{nt}$ & $(1500-2000) \mathrm{nt}$ & $\geq 2000 \mathrm{nt}$ \\
\hline Contig & $\begin{array}{c}1,417,688 \\
(100)\end{array}$ & $\begin{array}{c}1,397,239 \\
(98.56)\end{array}$ & $9006(0.64)$ & $3761(0.27)$ & $2017(0.14)$ & $1633(0.12)$ & $4031(0.28)$ \\
\hline Trinity assembly & $23,727(100)$ & 0 & 8159 (34.39) & 4254 (17.93) & $2668(11.24)$ & $2238(9.43)$ & 6407 (27) \\
\hline Unigene (TGICL) & $22,059(100)$ & 0 & $7936(35.98)$ & 4048 (18.35) & 2495 (11.31) & $2039(9.24)$ & $5540(25.11)$ \\
\hline
\end{tabular}

Table 5. Clean reads of RNA-Seq mapped onto unigenes in three groups (i.e., TA, TB, and TC) of Mortierella alpina based on bowtie2.

\begin{tabular}{ccccc}
\hline Sample & Total Read & $\begin{array}{c}\text { Total Read } \\
\text { Mapped (\%) }\end{array}$ & $\begin{array}{c}\text { Unique Read } \\
\text { Mapped (\%) }\end{array}$ & $\begin{array}{c}\text { Multi-Region } \\
\text { Mapped (\%) }\end{array}$ \\
\hline TA & $46,514,296$ & $43,589,424(93.71)$ & $39,026,470(83.90)$ & $4,562,954(9.81)$ \\
TB & $40,287,982$ & $37,644,954(93.44)$ & $33,595,404(83.39)$ & $4,049,550(10.05)$ \\
TC & $55,154,048$ & $51,442,932(93.27)$ & $45,945,988(83.30)$ & $5,496,944(9.97)$ \\
\hline
\end{tabular}

\subsection{Functional Annotation of Unigenes in Mortierella alpina}

The unigenes of $M$. alpina were annotated by a BLAST analysis based on the $\mathrm{Nr}$ database of the NCBI with an E-value threshold of $1 \times 10^{-5}$. A total of $10,224(46.35 \%$ of $22,059)$ unigenes were identified with putative homologues in the $\mathrm{Nr}$ database. The results revealed that a total of $5235(51.20 \%)$ of the matched sequences were highly homologous, with an E-value $<1 \times 10^{-5}$ (Table S3).

The functions of the unigenes were further annotated and classified using the GO database (Figure 1; Table S4). Among the 3344 unigenes annotated with GO terms, a total of 2718 (81.28\%) unigenes were classified in the category of molecular function, followed by the categories of biological processm with 2027 (60.62\%) unigenes and cellular component of 1266 (37.86\%) unigenes annotated, respectively. The unigenes assigned to molecular function were mainly associated with catalytic activities, with 1828 (54.67\%) unigenes and binding with 1535 (45.90\%) unigenes, respectively. The unigenes assigned to biological process were mainly linked to metabolic processes, with 1641 (49.07\%) unigenes and cellular process of $1616(48.33 \%)$ unigenes, respectively. In the category of the cellular component, the two largest groups of unigenes were annotated according to cells, with 1264 (37.80\%) unigenes and the cell parts of 1264 (37.80\%) unigenes annotated, respectively.

The functions of the unigenes of $M$. alpina were further classified according to the COG database (Figure 2; Table S5). The results showed that a total of 1549 unigenes were annotated into a total of 23 functional categories in the COG database. The top five categories with the most unigenes annotated included the general function prediction only (289 unigenes, 18.66\%), translation, ribosomal structure, and biogenesis (120 unigenes, $7.75 \%$ ), post-translational modification, protein turnover, chaperones (119 unigenes, 7.68\%), replication, recombination and repair (114 unigenes, $7.36 \%$ ), and amino acid transport and metabolism (86 unigenes, 5.55\%). The two least represented categories included cell motility ( 3 unigenes, $0.19 \%$ ) and chromatin structure and dynamics ( 8 unigenes, $0.52 \%$ ). 

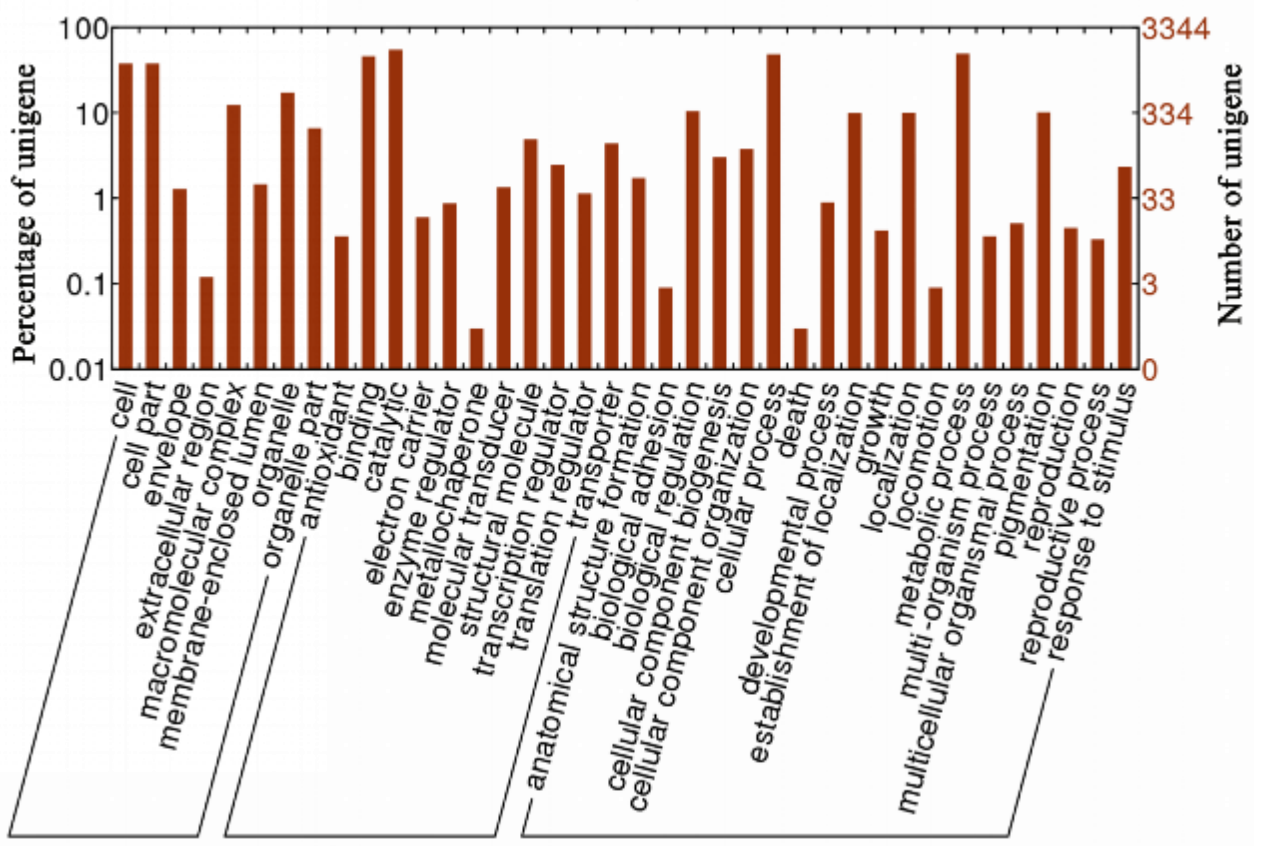

Cellular component Molecular function

Biological proces

Figure 1. Functional annotations of the unigenes of Mortierella alpina based on the Gene Ontology (GO) analysis.
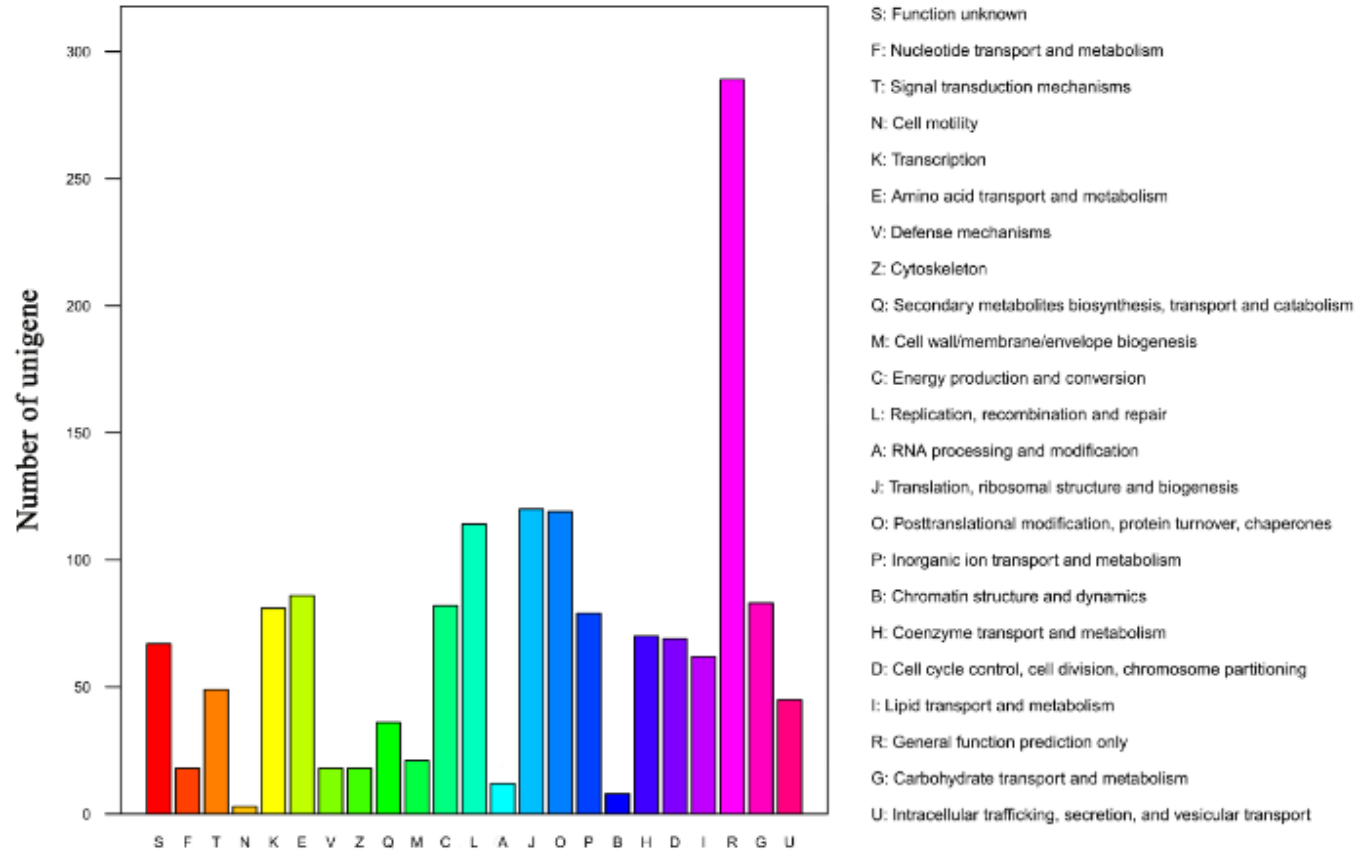

Figure 2. Functional annotations of the unigenes of Mortierella alpina based on the Cluster of Orthologous Groups (COG) database.

The unigenes of M. alpina were annotated with respect to metabolic pathways based on the KEGG database (Figure 3; Table S6). A total of 4302 unigenes were revealed in 330 metabolic pathways in the KEGG database. The top eight enriched metabolic pathways with the most unigenes annotated included the ribosome (190 unigenes, $4.42 \%$ ), the biosynthesis of amino acids (145 unigenes, 3.37\%), purine metabolism (124 unigenes, 2.88\%), carbon metabolism (119 unigenes, 2.77\%), oxidative phosphorylation (107 unigenes, $2.49 \%$ ), the spliceosome (107 unigenes, $2.49 \%$ ), RNA transport (102 unigenes, $2.37 \%$ ), and protein 
processing in the endoplasmic reticulum (101 unigenes, 2.35\%). In particular, the pathways closely associated with fatty acid metabolism included glycolysis/gluconeogenesis (57 unigenes, $1.32 \%$ ), glycerophospholipid metabolism (47 unigenes, $1.09 \%$ ), the citrate cycle (43 unigenes, $1.00 \%$ ), fatty acid degradation (35 unigenes, $0.81 \%$ ), sphingolipid metabolism (29 unigenes, $0.67 \%$ ), biosynthesis of unsaturated fatty acids (27 unigenes, $0.63 \%)$, the pentose phosphate pathway ( 26 unigenes, $0.60 \%$ ), glycerolipid metabolism (18 unigenes, $0.42 \%$ ), fatty acid elongation (12 unigenes, $0.28 \%$ ), and fatty acid biosynthesis (6 unigenes, $0.14 \%$ ).

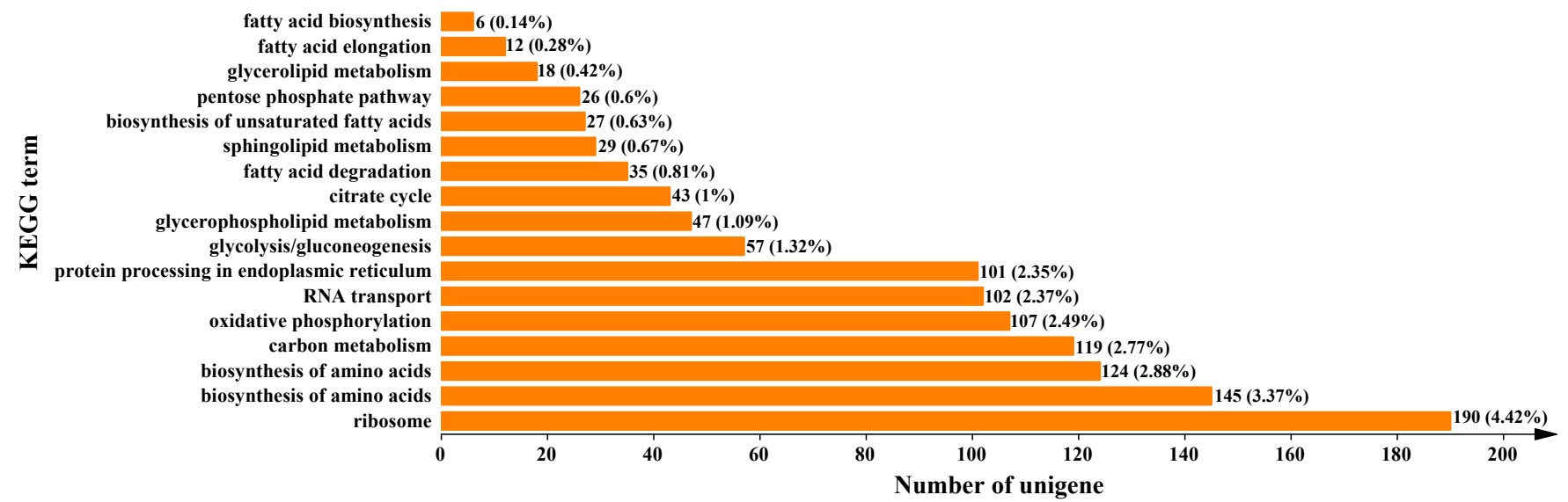

Figure 3. Functional annotations of the unigenes of Mortierella alpina based on the Kyoto Encyclopedia of Genes and Genomes (KEGG) database.

\subsection{Identification of Differentially Expressed Unigenes in Mortierella alpina}

To investigate the transcriptional variations in the three groups of M. alpina, DEGs were identified based on the pairwise comparisons among the three groups of $M$. alpina by comparing the FPKM values with a $p$-value $<0.05$ and fold changes $>2$ or $<-2$ (Figure 4A). A total of 1552 DEGs were revealed in the three pairwise comparison groups to be involved in the molecular response of M. alpina in response to cold stress. A total of 785, 853, and 710 DEGs were revealed in the pairwise comparisons of TA vs. TB, TA vs. TC, and TB vs. TC, with 332, 451, and 406 DEGs up-regulated and 453, 402 and 304 DEGs down-regulated, respectively (Figure $4 \mathrm{~B}$ ).

A

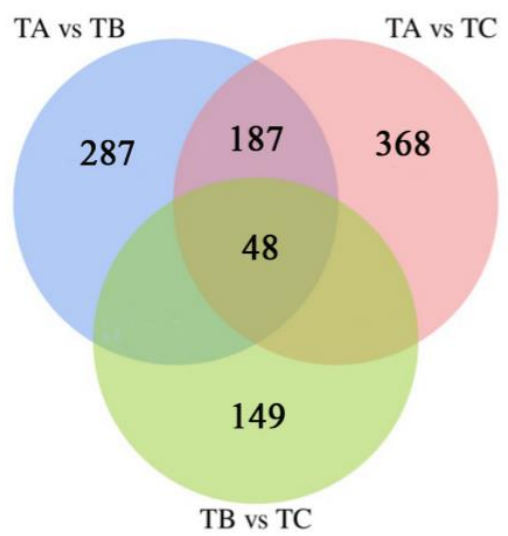

B

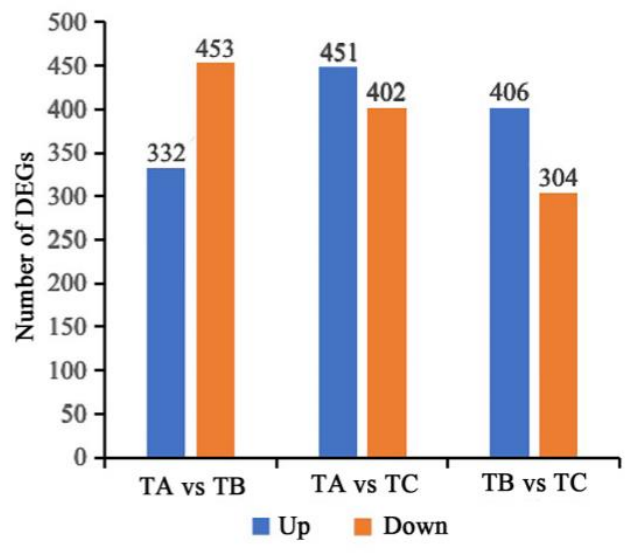

Figure 4. Differentially expressed genes (DEGs) identified in the pairwise comparisons among the three groups (i.e., TA, TB, and TC) of Mortierella alpina (A) showing up-regulated and down-regulated DEGs (B). 


\subsection{Enrichment Analysis of Differentially Expressed Genes Based on the Pairwise Comparisons of Mortierella alpina}

To further investigate the effects of cold stress on the gene expression of M. alpina, enrichment analyses based on both GO and KEGG databases were performed on the DEGs in the pairwise comparisons of the three groups of M. alpina. In the TA vs. TB comparison, the results of the GO annotations showed that the genes were mainly enriched in the functions of nucleotide binding, catalytic activity, pyridoxal phosphate binding, peptide activity, and autophagy (Figure 5A,B), while the KEGG enrichment analysis showed that the genes were mainly enriched in the cytosolic DNA-sensing pathway, carbon fixation in photosynthetic organisms, and protein digestion and absorption (Figure 5C).

In the TA vs. TC comparison, the GO annotations revealed that genes were mainly enriched in the oxidation-reduction process, proton-exporting ATPase activity, the phosphorylation mechanism, and metabolic processes (Figure 6A,B), while the KEGG enrichment analysis showed that genes were mainly enriched in the biosynthesis of unsaturated fatty acids, the PPAR signaling pathway, fatty acid degradation, peroxisomes, and alpha-linolenic acid metabolism (Figure 6C).

In the TB vs. TC group, the GO enrichment analysis showed that genes were mainly enriched with respect to proton-exporting ATPase activity, the phosphorylation mechanism, transfer activity, transferring acyl groups other than amino-acyl groups, the oxidationreduction process, metabolic processes, and the ATP biosynthetic process (Figure 7A,B), while the KEGG enrichment analysis showed that genes were mainly enriched in the pathways of peroxisomes, the PPAR signaling pathway, fatty acid biosynthesis, fatty acid degradation, and the biosynthesis of unsaturated fatty acids (Figure 7C).

Enrichment analyses of the 1552 DEGs revealed in the three groups of $M$. alpina were performed based on both the GO and KEGG databases. The results of GO enrichment analysis showed that these genes were mainly enriched in proton-exporting ATPase activity, the phosphorylation mechanism, the oxidation-reduction process, the ATP biosynthetic process, transferase activity, transferring acyl groups other than amino-acyl groups, and fatty acid biosynthetic process (Figure 8A,B), while the KEGG enrichment analysis revealed that genes were mainly enriched in the biosynthesis of unsaturated fatty acids, peroxisomes, the PPAR signaling pathway, fatty acid degradation, alpha-linolenic acid metabolism, primary bile acid biosynthesis, and fatty acid biosynthesis (Figure 8C).

Furthermore, the 48 DEGs shared among the three pairwise comparisons of M. alpina were annotated based on both the GO and KEGG databases. The results of the GO enrichment analysis showed that these genes were mainly enriched in macromolecule biosynthetic processes, isocitrate lyase activity, enoyl-(acyl-carrier-protein) reductase (NADH) activity, the fatty acid synthase complex, holo-(acyl-carrier-protein) synthase activity, and succinate dehydrogenase activity (Figure 9A,B), while the KEGG enrichment analysis showed that these genes were mainly enriched in the degradation of aromatic compounds, fatty acid biosynthesis, caprolactam degradation, alpha-linolenic acid metabolism, and steroid biosynthesis (Figure 9C).

\subsection{Verification of the Expression of Differentially Expressed Genes by qRT-PCR Analysis}

In order to verify the reliability of the expression patterns of DEGs revealed in the pairwise comparisons of M. alpina, six DEGs were randomly selected for further qRT-PCR analysis (Table 6). The results showed that the expressions of these DEGs were consistent with those revealed by the transcriptome sequencing results (Figure 10). 
A

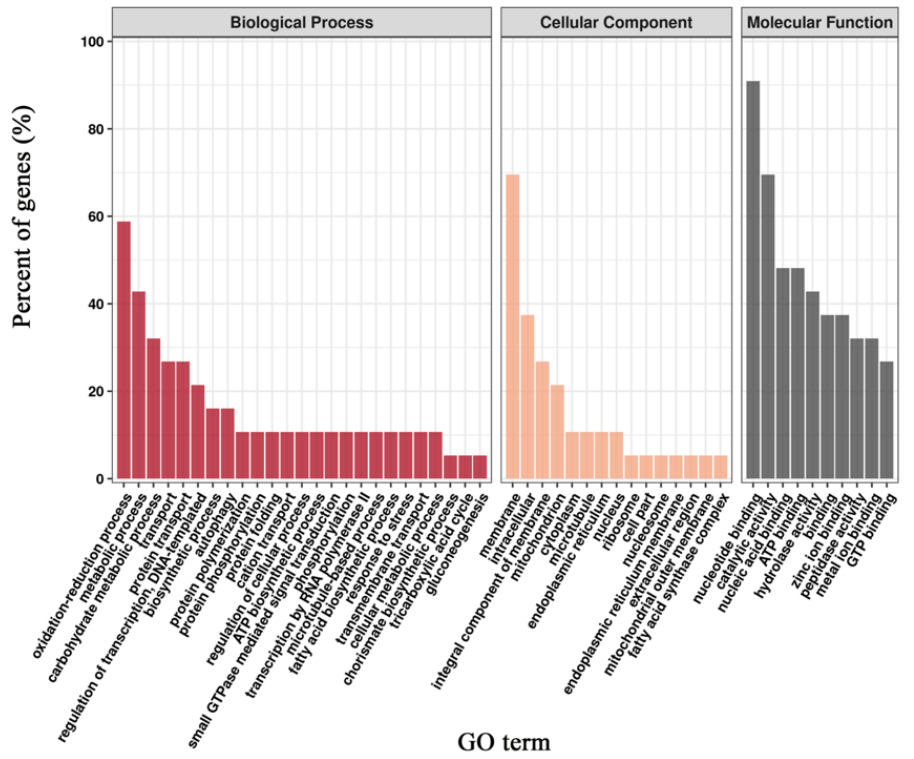

B

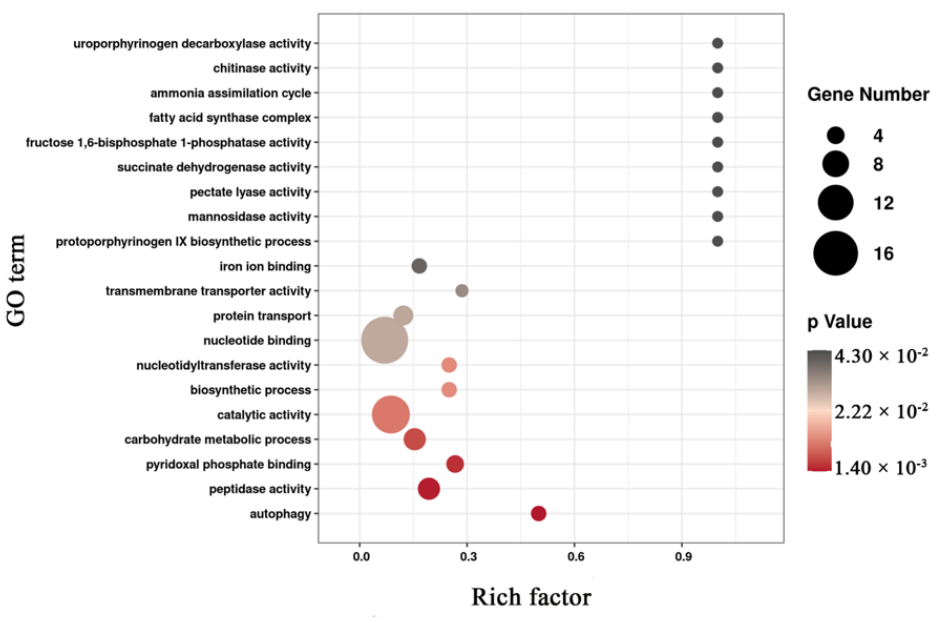

$\mathrm{C}$

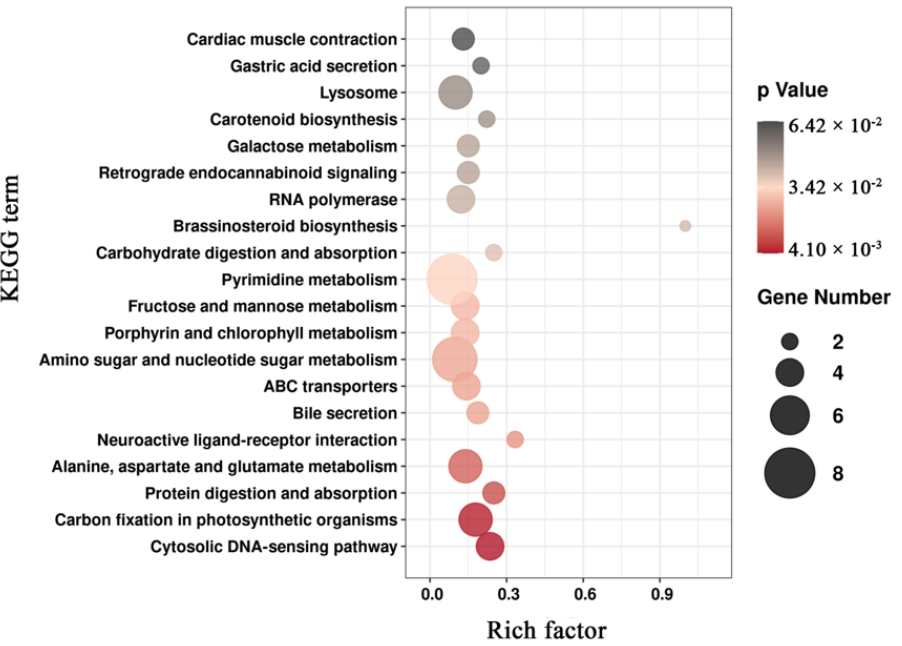

Figure 5. GO (A,B) and KEGG (C) enrichment analyses of differentially expressed genes (DEGs) derived from the TA vs. TB comparison of Mortierella alpina. 

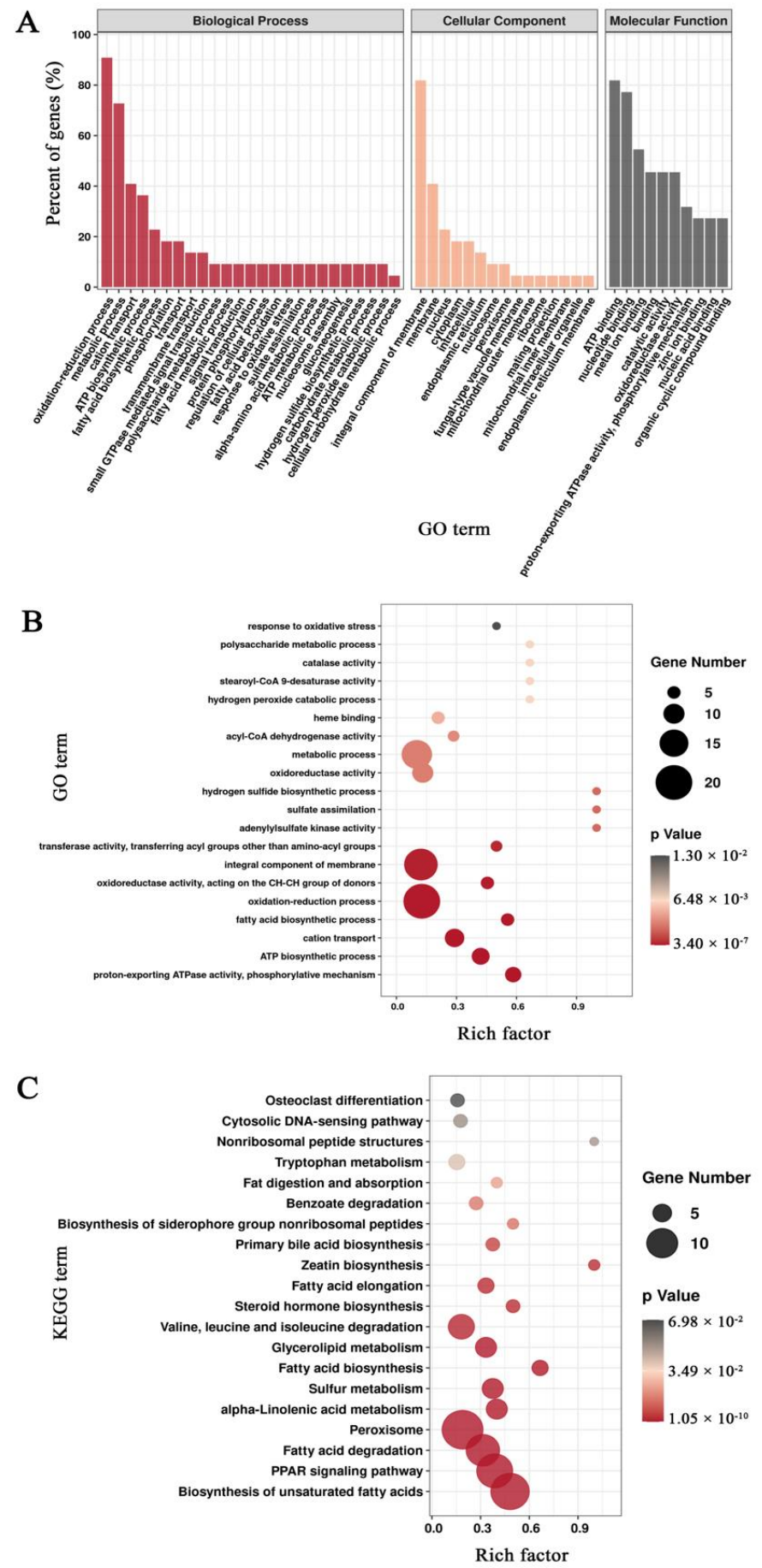

Figure 6. GO (A,B) and KEGG (C) enrichment analyses of differentially expressed genes (DEGs) derived from the TA vs. TC comparison of Mortierella alpina. 


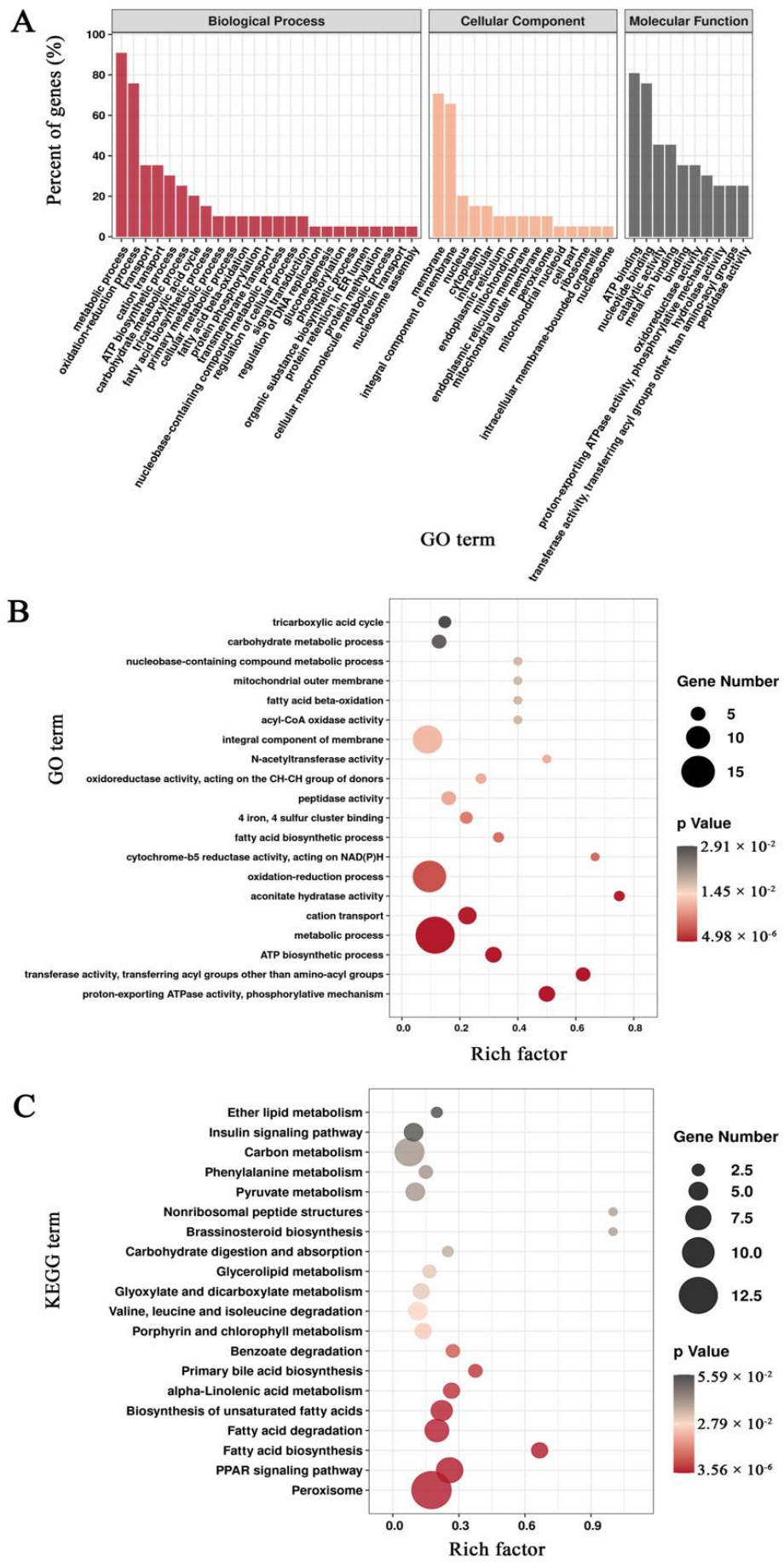

Figure 7. GO (A,B) and KEGG (C) enrichment analyses of differentially expressed genes (DEGs) derived from the TB vs. TC comparison of Mortierella alpina. 

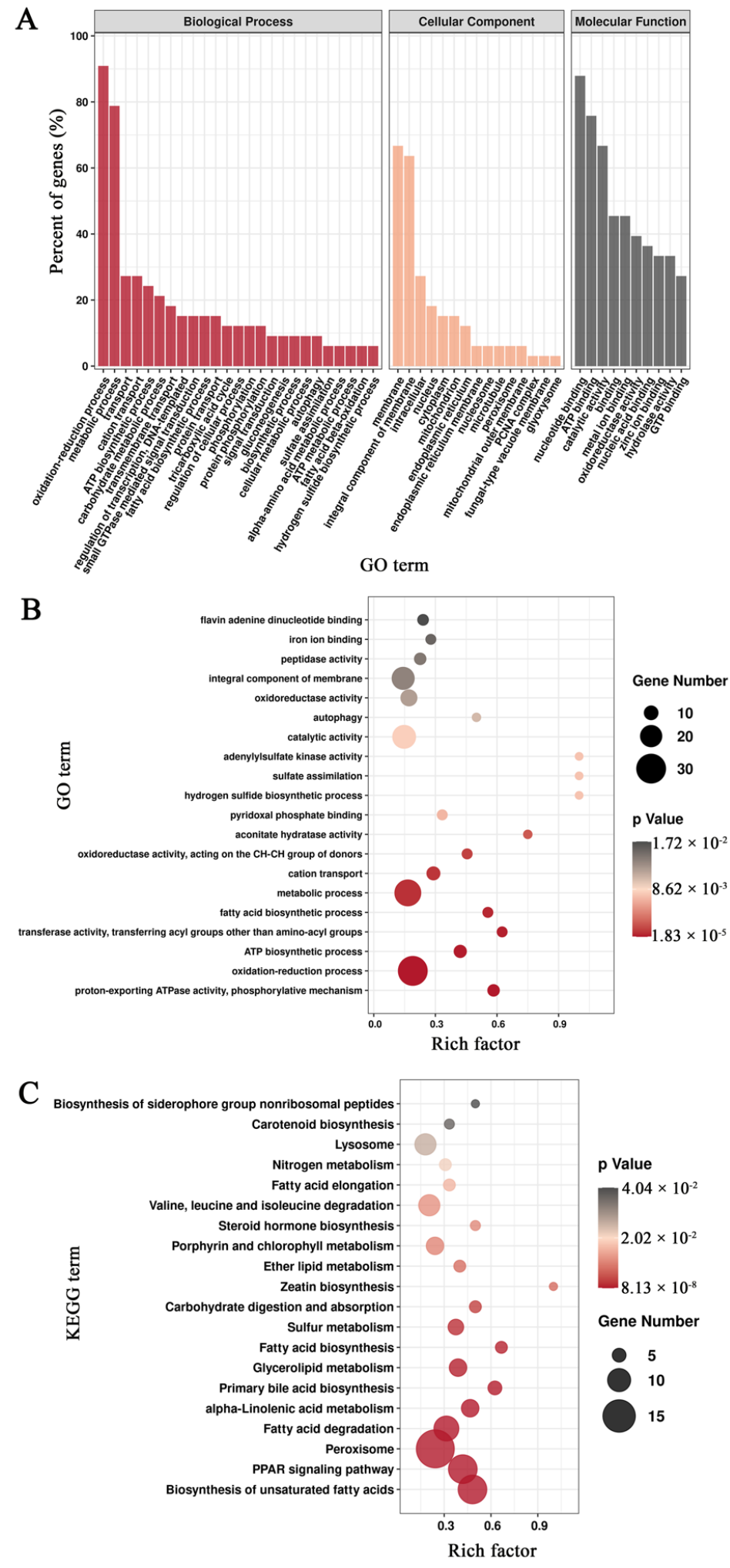

Figure 8. GO (A,B) and KEGG (C) enrichment analysis of differentially expressed genes (DEGs) derived from the three groups (i.e., TA, TB, and TC) of Mortierella alpina. 
A

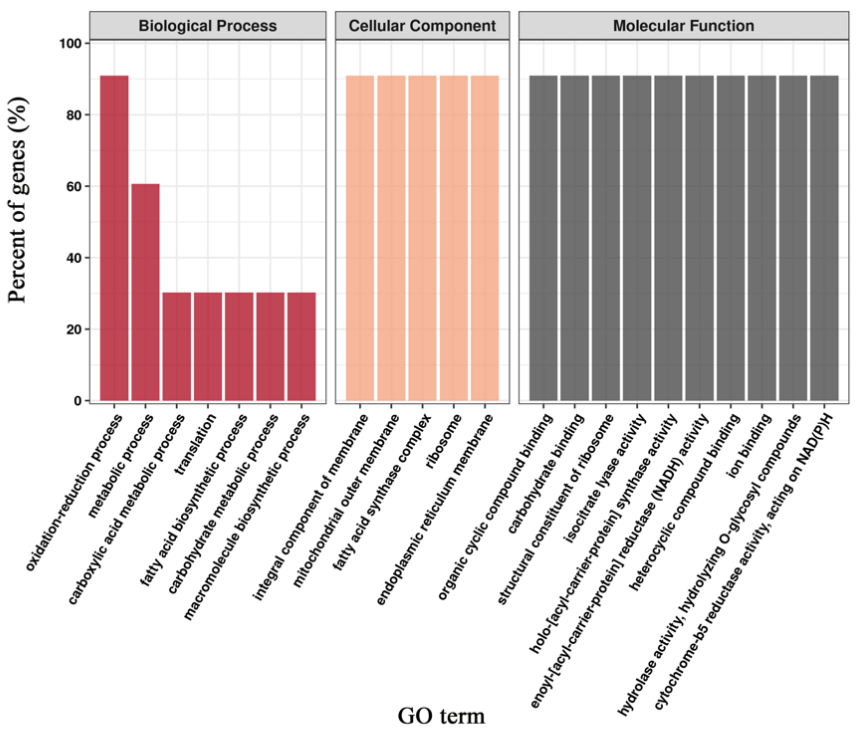

B

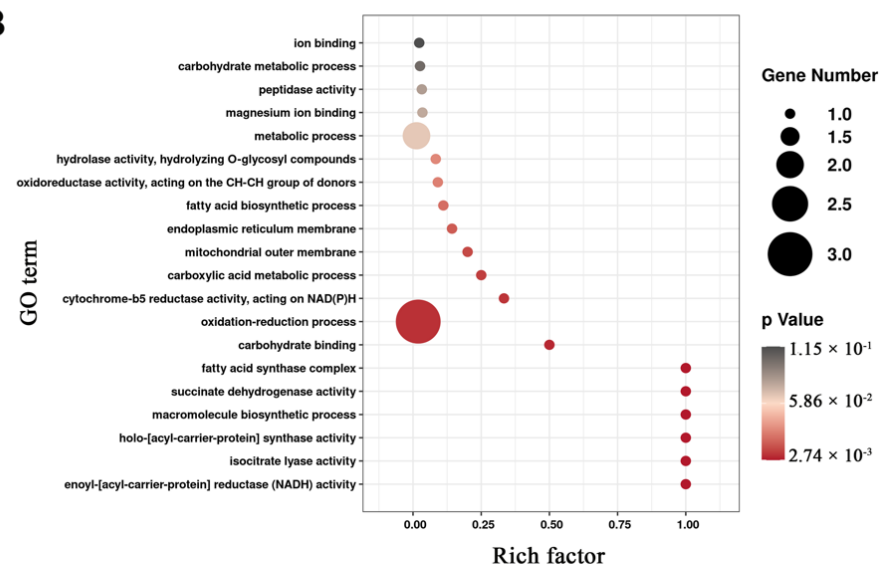

$\mathrm{C}$

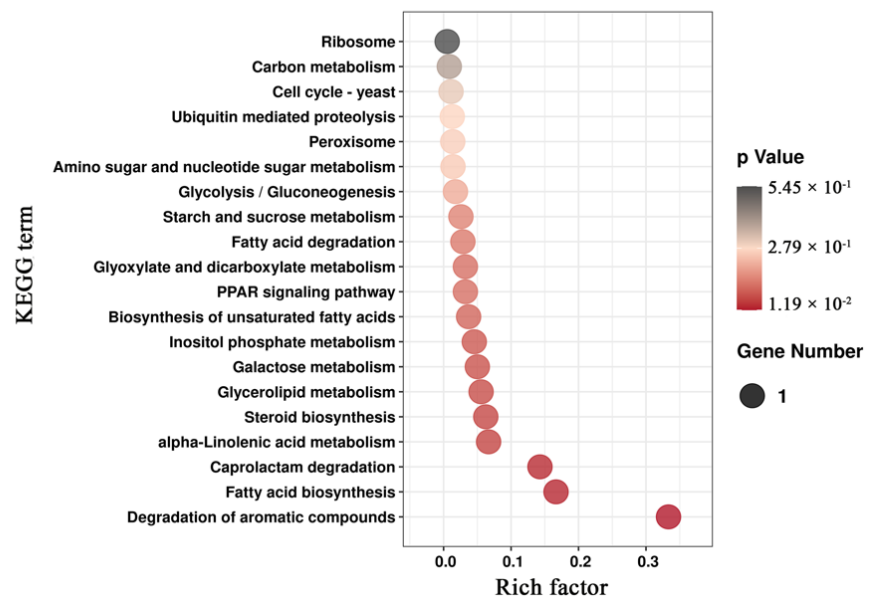

Figure 9. GO (A,B) and KEGG (C) enrichment analysis of differentially expressed genes (DEGs) shared among the three pairwise comparisons (i.e., TA vs. TB vs. TC) of the three groups of Mortierella alpina. 
Table 6. Expression profiles presented with values of the fragments per kilobase of transcript per million mapped reads (FPKM) of six differentially expressed genes (DEGs) of three groups of Mortierella alpina (TA, TB, and TC) randomly selected for the qRT-PCR analysis. Gene annotation is based on the non-redundant $(\mathrm{Nr})$ database at the National Center for Biotechnology Information (NCBI).

\begin{tabular}{|c|c|c|c|c|}
\hline Gene & Gene Annotation & TA & TB & TC \\
\hline c6160_g1_i1 & Sterol desaturase & 1.38 & 0.57 & 2.66 \\
\hline c11974_g1_i1 & Delta- 6 fatty acid desaturase & 28.3 & 35.2 & 39.27 \\
\hline c13286_g1_i1 & Delta-5 fatty acid desaturase & 552.74 & 287.16 & 287.03 \\
\hline$c 14468 \_g 1 \_i 1$ & Delta-6 fatty acid elongase & 885.54 & 790.17 & 473.17 \\
\hline c11922_g1_i1 & OPT oligopeptide transporter & 30.2 & 332.6 & 61.63 \\
\hline$c 12117 \_g 1 \_i 1$ & Isocitrate lyase & 74.24 & 4.76 & 383.25 \\
\hline
\end{tabular}

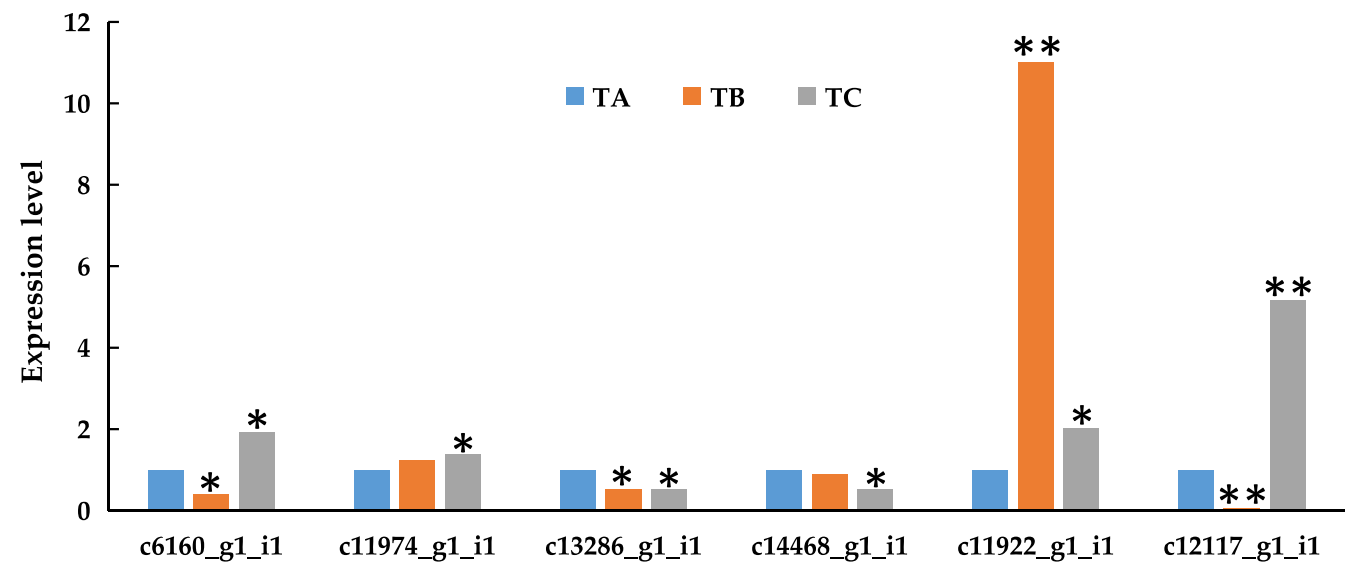

Figure 10. The qRT-PCR analysis of the expression patterns of six differentially expressed genes (DEGs) revealed in the three groups (i.e., TA, TB, and TC) of Mortierella alpina. Symbols "*" and "***" indicate the significant difference set at $p$ values less than 0.05 and 0.01 , respectively, in comparison to TA.

\subsection{Expression of Genes Involved in the Synthesis of Polyunsaturated Fatty Acids}

The main enzymes involved in the synthesis of PUFAs in $M$. alpina included $\Delta-9$ desaturase, $\Delta-12$ desaturase, $\Delta-6$ desaturase, $\Delta-5$ desaturase, $\omega-3$ desaturase, and fatty acid elongase, involved in fatty acid chain extension (Table S7). Our results revealed a total of three copies of genes encoding the $\Delta-9$ desaturase, three copies of genes encoding the $\Delta-6$ desaturase, one copy of gene each encoding $\Delta-12$ desaturase and $\Delta-5$ desaturase, and a total of four copies of genes encoding the fatty acid elongases. One copy of the gene encoding $\omega-3$ desaturase was identified with relatively small FPKM values. Compared with the TA group, the expressions of genes encoding the fatty acid desaturases were significantly down-regulated in the TB group. Two of the three copies of genes encoding the $\Delta-9$ desaturase (i.e., $\Delta-9-1$ and $\Delta-9-2$ ) showed similar expression levels, while the third copy (i.e., $\Delta$-9-3) showed significantly increased expression in the TA group, 11.42 times higher than that in the TB group. One of the three copies of genes encoding $\Delta-6$ desaturase (i.e., $\Delta-6-1$ ) showed similar expression levels between the TA and TB groups, while the other two copies (i.e., $\Delta-6-2$ and $\Delta-6-3$ ) showed increased expression levels, 2.18 and 3.23 times higher than those in the TA group and TB group, respectively. Furthermore, the expression levels of genes encoding $\Delta-12$ desaturase and $\Delta-5$ desaturase in the TA group were 2.31 and 1.92 times higher than those in the TB group, respectively, while the expression of gene encoding $\omega-3$ desaturase in the TB group was increased by 1.69 times compared to the TA group. Compared with the TA group, the expression of genes encoding the fatty acid elongase were slightly up-regulated in the TB group. 


\section{Discussion}

In this study, the transcriptomic analyses of three groups of $M$. alpina under three different temperature settings were conducted to investigate the molecular response to cold stress in this fungal species. The results of enrichment analyses based on both the GO and KEGG databases and the GC-MS analysis revealed the effects of cold stress on the expression of genes involved in lipid production in M. alpina. Specifically, M. alpina cultured under cold stress showed reduced growth and development due to an altered fundamental metabolism, i.e., less effective utilization of glucose and reduced activities of enzymes involved in the metabolic pathway of glycerol, ultimately leading to a decrease in the synthesis of glycerol, triglycerides, and fatty acids. Our results showed that the lipid contents were reduced by approximately 1.78 -fold under cold stress (Table 1). Furthermore, the cold stress caused variations in the carbon metabolism-related pathways of M. alpina, which generated both acetyl-CoA and NADPH. Acetyl-CoA is an important substrate and precursor for fatty acid synthesis, while NADPH is a reducing agent in the process of fatty acid synthesis. The reduction of acetyl-CoA and NADPH contents led to a decrease in the lipid contents of M. alpina. Moreover, cold stress caused variations in the expressions of genes involved in fatty acid synthesis in M. alpina, ultimately changing the fatty acid composition of $M$. alpina. Our results constantly showed that cell membranes were the most significantly enriched GO terms in the category of cellular components based on the GO database and the locations of the most significantly enriched metabolic pathway based on the KEGG database (Figures 5-9), suggesting that cold stress had the greatest impact on the membrane structure of M. alpina. As the structural components of cell membranes, lipid storage, and signal transduction pathways, long-chain PUFAs play a key role in higher organisms. These results suggested that cold stress altered the fatty acid composition in M. alpina, indirectly changing the expression of genes involved in cell membranes, lipid storage, and signal transduction pathways.

We further discuss the molecular regulations of genes involved in the metabolic pathways closely related to lipid production and composition in M. alpina under cold stress.

\subsection{Fatty Acid Metabolic Pathway}

The metabolic pathway of PUFAs in M. alpina under cold stress was described in Figure 11. A total of three copies of genes encoding $\Delta-9$ desaturase and four copies of genes encoding the fatty acid elongase were identified in our study (Table S7). The $\Delta-9$ desaturase catalyzed the conversion of stearic acid (C18:0) to generate the monounsaturated fatty acid OA (C18:1), which was the first step in unsaturated fatty acid synthesis in $M$. alpina [31,32]. Studies have shown that the overexpression of two copies of the genes (i.e., $\Delta-9-1$ and $\Delta-9-2$ ) encoding the $\Delta-9$ fatty acid desaturase in Saccharomyces cerevisiae indicated that $\Delta-9-1$ and $\Delta-9-2$ were more likely to catalyze oleic acid, instead of palmitic acid, to produce stearic acid [33]. Furthermore, the other copy of the gene encoding $\Delta-9$ desaturase (i.e., $\Delta$-9-3) was homologously expressed in $M$. alpina, showing that at a low temperature $\left(12{ }^{\circ} \mathrm{C}\right), \Delta-9-3$ enhanced the conversion of palmitic acid (C16:0) to palmitoleic acid (C16:1) by 9.12 times [34]. However, the formation of C16:1 was not detected by the GC-MS analysis in our study, probably due to the relatively high temperature of $15^{\circ} \mathrm{C}$ in comparison to $12{ }^{\circ} \mathrm{C}$. Our results also revealed three copies of genes encoding the $\Delta$ 6 desaturase and one copy of each gene encoding $\Delta-12$ desaturase and $\Delta-5$ desaturase, respectively. The $\Delta-12$ desaturase converted oleic acid to generate linoleic acid, while the $\Delta-6$ desaturase converted linoleic acid to $\gamma$-linolenic acid. Studies have shown that the overexpression of both $\Delta-12$ desaturase and $\Delta-6$ desaturase in Saccharomyces cerevisiae caused the increased production of $\gamma$-linolenic acid by $10 \%$ in total fatty acids [35]. The $\Delta-5$ desaturase promoted the conversion of eicosatrienoic acid to ARA and the conversion of ARA to EPA. Studies have shown that the overexpression of genes encoding the $\Delta-5$ desaturase in Arachis hypogaea caused the production of EPA [36]. In our study, the results of the transcriptome analysis showed that, under cold stress, the expression levels of $\Delta-9$ desaturase, $\Delta-12$ desaturase, $\Delta-6$ desaturase, and $\Delta-5$ desaturase decreased, leading to the 
down-regulation of the production of oleic acid, linoleic acid, $\gamma$-linolenic acid, and ARA in $M$. alpina. Furthermore, our results showed that the expression of $\omega-3$ desaturase was up-regulated in M. alpina under cold stress. Studies have shown that, at room temperature, $\omega-3$ desaturase hardly used C20 $\omega-6$ fatty acids but only C18 $\omega-6$ fatty acids as substrates to generate $\omega-3$ fatty acids [37]. These results were consistent with the findings reported in our current study, showing that EPA (i.e., C20:5 $\omega-3$ unsaturated fatty acid) was hardly produced at a normal temperature of $25^{\circ} \mathrm{C}$, whereas a small amount of EPA was detected in $M$. alpina under the low temperature of $15^{\circ} \mathrm{C}$. Moreover, our results also showed that cold stress led to the up-regulation of genes encoding two fatty acid elongases, i.e., elongase 1 , converting palmitic acid to stearic acid, and elongase 2, converting C18:3 n-6 to C20:3 n-6. These results were consistent with the effects of cold stress on the fatty acid composition of M. alpina (Table 2), showing that the contents of stearic acid and C20:3 n-6 were increased, while the content of C18:3 n-6 was decreased in M. alpina under cold stress.

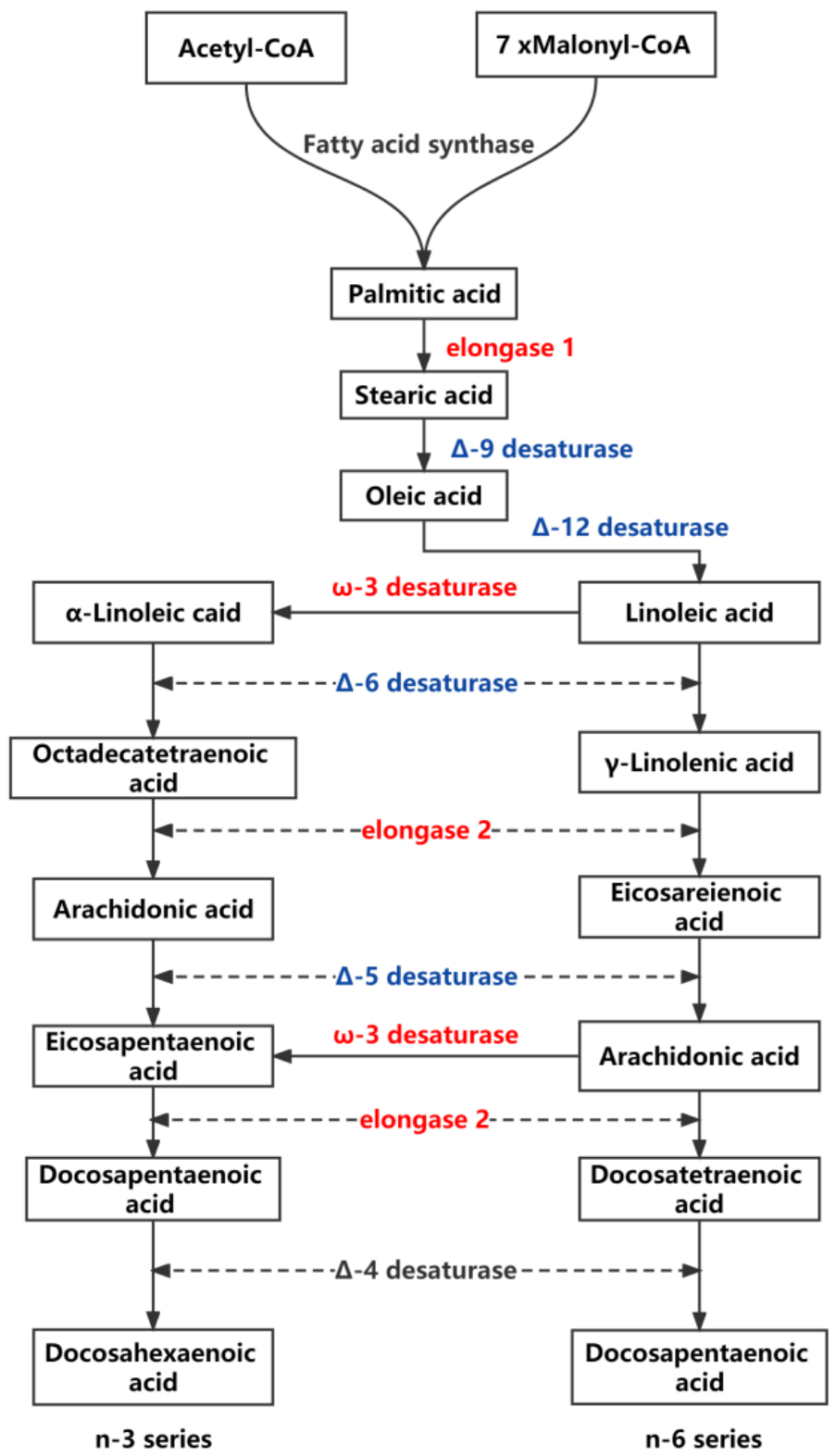

Figure 11. Metabolic pathway of polyunsaturated fatty acids (PUFAs) in Mortierella alpina under cold stress. Enzymes showing up-regulation and down-regulation are indicated in red and blue, respectively. 


\subsection{Glycerolipid and Glycerophospholipid Metabolic Pathways}

Studies have shown that glycerolipids (GLs) and glycerophospholipids (GPLs) account for high proportions of lipid composition in M. alpina-89\% and 8\%, respectively [7]. The GLs are mainly stored in lipid droplets, existing in the form of triacylglycerol. In our study, the transcriptomic results revealed a large number of variations in the expression levels of the genes involved in the GL and GPL pathways in M. alpina under cold stress lasting either 7 or 2 days (Figure 12; Table S7). These transcriptomic results suggested the following molecular mechanisms of $M$. alpina in response to cold stress at two different stages. In the early stage of cold stress, the low temperature caused a decrease in membrane fluidity in M. alpina, while the expressions of genes encoding alcohol dehydrogenase and glycerol kinase were increased. The precursor of glycerol was used to generate glycerol, which was then converted to 1-Acyl-sn-glycerol-3P. Although the down-regulation of the gene encoding 1-Acyl-sn-glycerol-3-phosphate acyltransferase caused the decreased conversion of 1,2-Diacyl-sn-glycerol-3P, the increased production of 1-Acyl-sn-glycerol-3P contributed to the increased production of 1,2-Diacyl-sn-glycerol-3P, catalyzed by adipose triglyceride lipase. As the precursors of phosphatidylinositol, phosphatidyicholine, phosphatidylserine, and phosphatidylethanolamine, the increased production of 1,2-Diacyl-sn-glycerol-3P ultimately increased the contents of GPLs in M. alpina. As the main structural components of the cell membrane, the GPLs are responsible for maintaining plasma membrane fluidity. Studies have shown that, at low temperatures, increased desaturation in membrane lipids is involved in the self-protection mechanism in M. alpina [33]. In the later stage of cold stress, the effects of cold stress on the fundamental metabolism of M. alpina were already evident, i.e., the rate of producing precursor substances was decreased, while the accumulated precursor substances were still constantly consumed. Furthermore, the expression of genes involved in the GL pathway were down-regulated, causing decreased production of 1,2-diacyl-sn-glycerol-3P and reduced synthesis of GPLs. Furthermore, the expressions of genes encoding the alkali O-acetyl transferase and the triglycerol fat enzyme were decreased to ultimately prevent the degradation and oxidation of fatty acids in M. alpina under cold stress.

\subsection{Acetyl-CoA and NADPH Metabolism}

As one of the important types of starting materials for fatty acid synthesis [38], acetyl$\mathrm{CoA}$ is generally produced in one of two ways in M. alpina. First, the pyruvate is catalyzed by both the mitochondrial pyruvate dehydrogenase complex and the citrate synthase to produce citrate, which is cleaved by ATP:citrate lyase to generate acetyl-CoA [39]. Our results showed that the genes encoding hexokinase (EC: 2.7.1.1; FPKM values increased from 211.68 in th TA group to 384.34 in the TB group), 3-phosphoglycerate kinase (EC: 2.7.2.3; FPKM values increased from 205.51 in the TA group to 279.56 in the TB group), fructose-bisphosphate aldolase (EC: 4.1.2.13; FPKM values increased from 0 in the TA group to 0.36 in the TB group), and pyruvate kinase (EC: 2.7.1.40; FPKM values increased from 268.38 in the TA group to 423.91 in the TB group) were up-regulated in M. alpina under cold stress, ultimately increasing the production of both pyruvate and acetyl-CoA (Figure 13; Table S7). Furthermore, the expression of the gene encoding lactate dehydrogenase (EC: 1.1.1.27; FPKM values increased from 489.81 in the TA group to 2359.39 in the TB group), which catalyzes the production of pyruvate from lactic acid, was increased significantly to enhance the synthesis of pyruvate. Moreover, the expression of the gene encoding the phosphoenolpyruvate kinase (EC: 4.1.1.49; FPKM value decreased from 169.83 in the TA group to 126.17 in the TB group), which catalyzes the conversion of oxaloacetate to phosphoenolpyruvate, was decreased, ultimately decreasing the consumption of pyruvate and increasing the production of acetyl-CoA, catalyzed by pyruvate dehydrogenase (EC: 2.3.1.12). Second, acetyl-CoA is generated by the oxidation of both fatty acids and amino acids in the mitochondria [39]. Our enrichment analysis results based on the KEGG database showed that, under cold stress, the DEGs in M. alpina were significantly enriched in the pathway of fatty acid degradation. These results were consistent with those reported 
previously, showing that the acetyl-CoA and energy produced by fatty acid degradation played the important structural and regulatory roles in fatty acid synthesis [40].

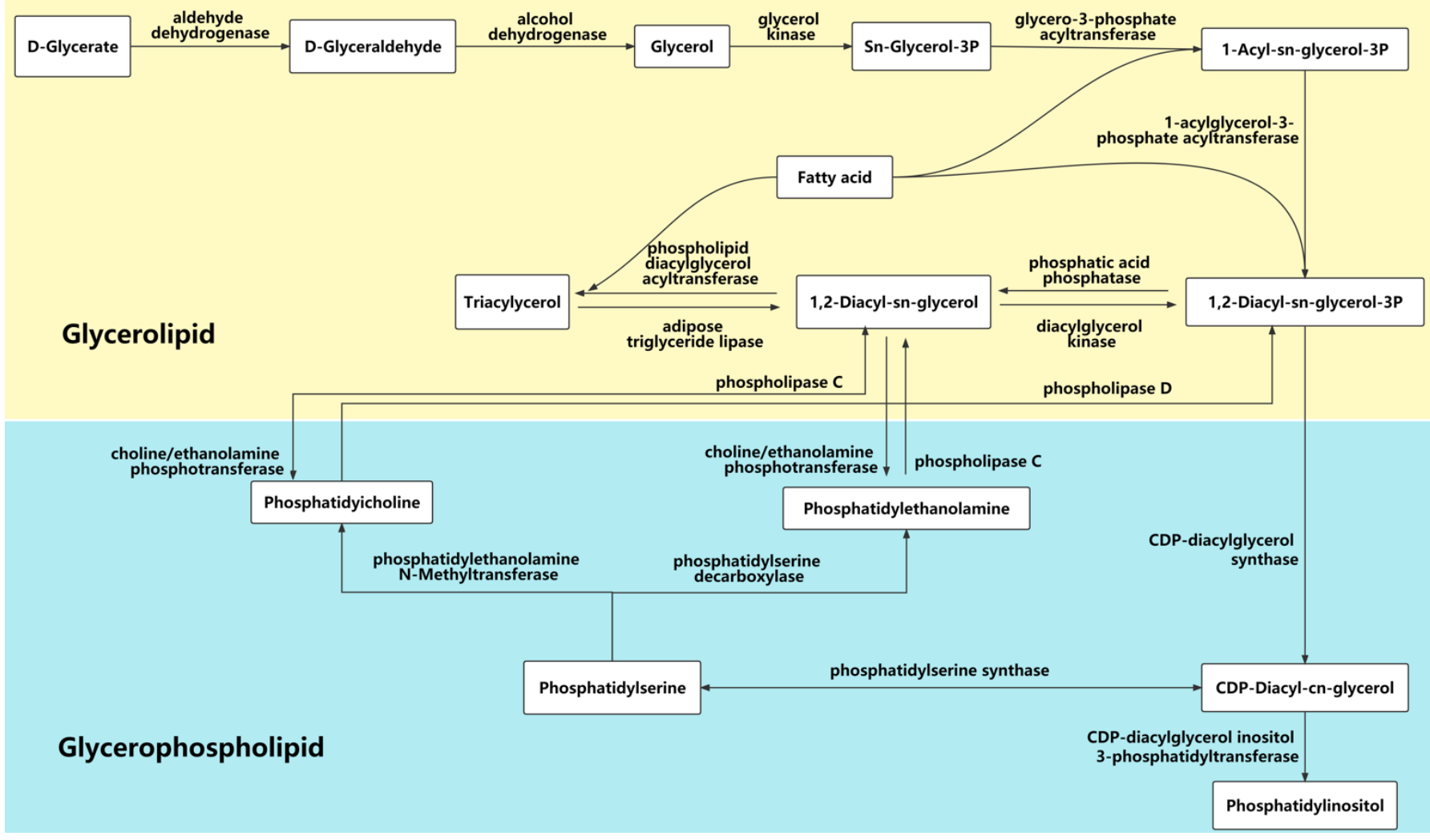

Figure 12. Metabolic pathways of glycerolipids and glycerophospholipids.

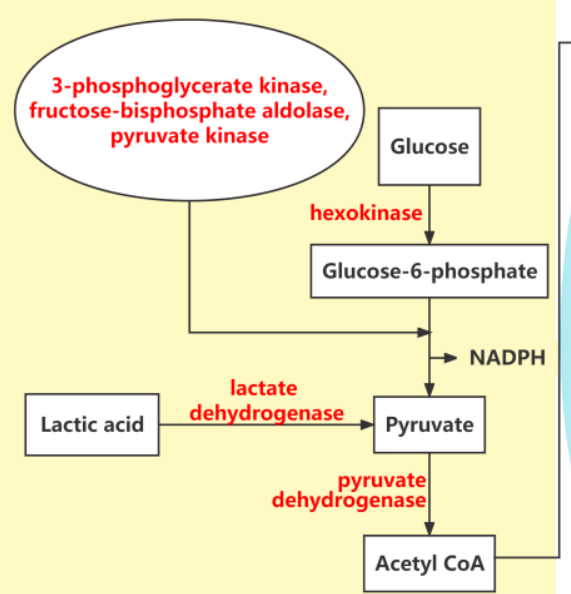

Glycolytic pathway

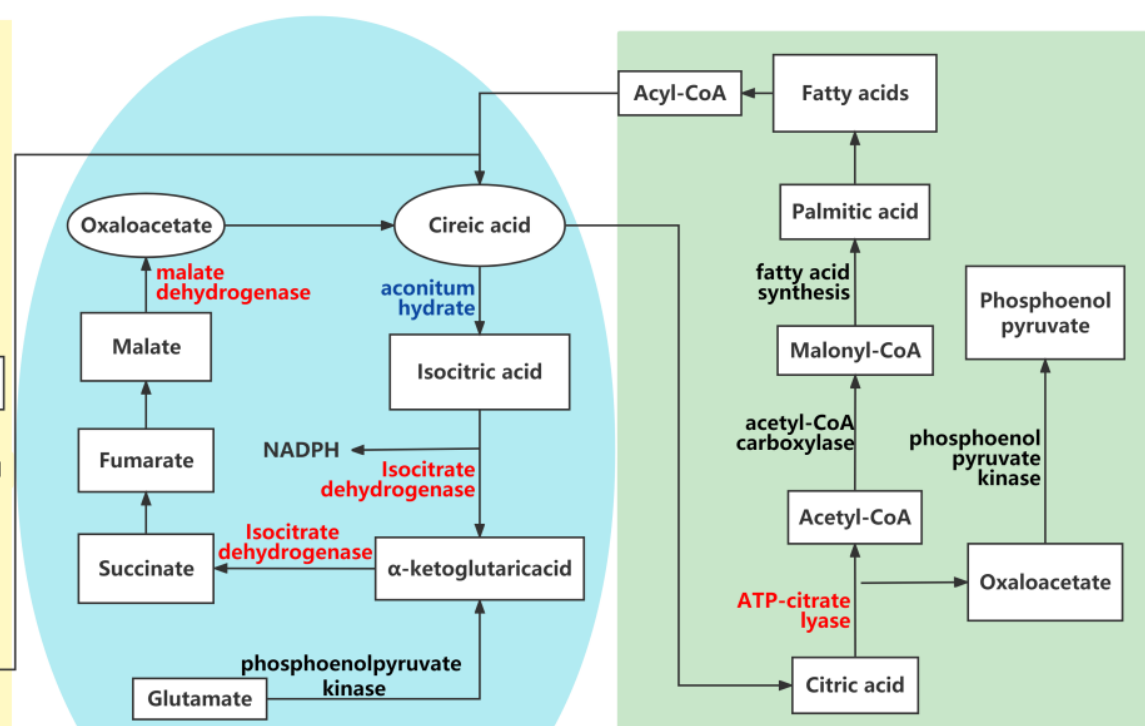

Tricarboxylic acid cycle
Biosynthesis of fatty acids

Figure 13. Acetyl-CoA and NADPH metabolism involved in the glycolytic pathway, the tricarboxylic acid (TCA) cycle, and the biosynthesis of fatty acids. The up-regulated and down-regulated enzymes are highlighted in red and blue, respectively. 
As the key reducing agent in many metabolic pathways, NADPH is also a key cofactor for fatty acid synthesis and desaturation [39]. However, it is not clear how NADPH is mainly generated in M. alpina. NADPH is generally produced in multiple ways in microorganisms. First, NADPH is generated in the TCA cycle for the synthesis of fatty acids as well as citric acid. Our results showed that the expression level of the gene encoding citrate lyase (EC: 2.3.3.8; FPKM values increased from 1109.95 in the TA group to 1461.35 in the TB group), which catalyzes citric acid to produce acetyl-CoA, was increased in $M$. alpina under cold stress (Figure 13; Table S7). Furthermore, the expression of the gene encoding aconitum hydratase (EC: 4.2.1.3; FPKM values decreased from 171.67 in the TA group to 112.03 in the TB group) was decreased, reducing the conversion of citric acid to isocitrate, while the expression of the gene encoding isocitrate dehydrogenase (EC: 1.1.1.41; FPKM values increased from 276.03 in the TA group to 370.08 in the TB group) was increased to convert isocitrate to oxalosuccinate and to produce NADPH. Second, NADPH is also generated in the pentose phosphate pathway and the malate/pyruvate cycle in M. alpina. For example, as the main provider of NADPH in oleaginous microorganisms, the overexpression of the gene encoding the malic enzyme (ME) in Mucor circinelloides caused the increased activity of ME and increased lipid contents, suggesting that the production of NADPH catalyzed by ME was an important rate-limiting step in fatty acid synthesis [41]. Furthermore, the effects of glucose-6-phosphate dehydrogenase (G6PD), 6-phosphogluconate dehydrogenase (PGD), and ME in lipid synthesis were investigated in $M$. alpina by transcriptomic analysis, showing that inhibition of G6PD resulted in the reduction of $\sim 40 \%$ in NADPH levels and a significant reduction in fatty acid content, with lesser effects of knockdowns of both PGD and ME on NADPH levels and fatty acid accumulation, suggesting that NADPH produced by the pentose phosphate pathway played a crucial role in lipid synthesis [9]. These results were consistent with the findings revealed in our study. Our results showed that the expression of genes encoding malate dehydrogenase (EC: 1.1.1.37; FPKM values increased from 414.72 in the TA group to 559.21 in the TB group) and citrate synthase (EC: 2.3.3.1; FPKM values increased from 141.16 in the TA group to 167.71 in the TB group) were increased slightly to enhance the synthesis of citric acid. Moreover, the expressions of genes encoding glutamate dehydrogenase (EC: 1.4.1.3; FPKM values increased from 61.01 in the TA group to 112.89 in the TB group) and pyruvate dehydrogenase (EC: 2.3.1.12, FPKM values increased from 108.92 in the TA group to 141.65 in the TB group) were increased, with the former converting glutamic acid into $\alpha$-ketoglutarate and generating NADPH and the latter converting pyruvate into acetylCoA. It was noted that the expression of the gene encoding ME (EC: 1.1.1.40; FPKM values decreased from 246.91 in the TA group to 33.79 in the TB group) decreased, indicating that malic acid was first converted to oxaloacetic acid, instead of pyruvate, and then citric acid.

Agricultural microorganisms have been used widely to produce microbial food, with the synthesis and metabolism of fatty acids extensively investigated. In particular, the metabolic pathway of fatty acid metabolism in M. alpina is well explored and unsaturated fatty acids are produced in $M$. alpina for the production of microbial food. It is expected that our study of the effect of low temperature on the regulation of the pathway of fatty acid metabolism in M. alpina could provide novel guidance for the study of fatty acid metabolism in other agricultural microorganisms to enhance the development of the agricultural food industry. Future experiments are necessary to identify the cold-dependent traits and to further verify the expression patterns of genes revealed in our study to further explore the molecular mechanisms regulating the response to cold stress in M. alpina.

\section{Conclusions}

Cold stress altered fundamental metabolism to decrease the growth and development of $M$. alpina. The results of a transcriptomic analysis showed that cold stress regulated the expressions of genes encoding the enzymes involved in the glycolysis pathway, fatty acid synthesis, the TCA cycle, the pentose phosphate pathway, the glycerolipid pathway, and the glycerophopholipid pathway, leading to the increased production of both acetyl-CoA and 
NADPH and increased synthesis of myristic acid (C14:0) and palmitic acid (C16:0). Cold stress caused the increased expression of the genes encoding $\omega-3$ desaturase and enhanced the conversion of ARA to EPA, while the expression of genes encoding the other desaturases (i.e., $\Delta-9$ desaturase, $\Delta-12$ desaturase, $\Delta-6$ desaturase, and $\Delta-5$ desaturase) decreased, leading to the accumulation of stearic acid (C18:0) and decreased production of other types of fatty acids such as the oleic acid (C18:1), linoleic acid (C18:2), and eicosatrienoic acid (C20:3). The expression patterns of six randomly selected DEGs revealed in the transcriptomic analysis were further verified by qRT-PCR analysis, indicating the high reliability of the RNA-Seq analysis. Our findings provide novel evidence to support the investigation of the molecular mechanisms of M. alpina in response to cold stress and to further explore lipid metabolism in M. alpina, ultimately facilitating the development of novel fermentation strategies to improve lipid production in M. alpina.

Supplementary Materials: The following supporting information can be downloaded at: https: / / www.mdpi.com/article/10.3390/agronomy12030599/s1, Table S1: Quality of RNA in samples of Mortierella alpina determined with a Nanodrop2000 micro UV-Vis spectrophotometer; Table S2: Primers and primer sequences used in the quantitative real-time PCR (qRT-PCR) analysis; Table S3: Annotation of unigenes in Mortierella alpina based on the non-redundant ( $\mathrm{Nr}$ ) database of the National Center for Biotechnology Information; Table S4: Annotation of unigenes in Mortierella alpina based on the Gene Ontology (GO) database; Table S5: Annotation of unigenes in Mortierella alpina based on the Cluster of Orthologous Groups (COG) database; Table S6: Annotation of unigenes in Mortierella alpina based on the Kyoto Encyclopedia of Genes and Genomes (KEGG) database; Table S7: Gene expression in Mortierella alpina based on the fragments per kilobase of transcript per million mapped reads (FPKM) method.

Author Contributions: Conceptualization, D.L. and Z.G.; methodology, D.L.; software, C.M.; validation, J.R., X.Z. and Z.W.; formal analysis, J.R.; investigation, H.W.; resources, D.L.; data curation, H.W.; writing — original draft preparation, J.R.; writing — review and editing, F.S.; visualization, J.R.; supervision, D.L.; project administration, Z.G.; funding acquisition, D.L. and Z.G. All authors have read and agreed to the published version of the manuscript.

Funding: This research was funded by the National Key R\&D Program of the Ministry of Science and Technology of China (2019YFA0904900), the National Natural Science Foundation of China (31972815 and 42176124), the Natural Science Foundation of Shandong Province (ZR2019ZD17, ZR2020ZD23, and ZR2021MC051), the scientific research fund of Binzhou Medical University (BY2021KYQD25 and BY2021KYQD28), and the Open Fund of Shandong Provincial Key Laboratory of Plant Stress (SPKLPS202001). The APC was funded by ZQG.

Conflicts of Interest: The authors declare no conflict of interest. The funders had no role in the design of the study; in the collection, analyses, or interpretation of data; in the writing of the manuscript, or in the decision to publish the results.

\section{References}

1. Ji, X.J.; Ren, L.J.; Nie, Z.K.; Huang, H.; Ouyang, P.K. Fungal arachidonic acid-rich oil: Research, development and industrialization. Crit. Rev. Biotechnol. 2014, 34, 197-214. [CrossRef]

2. Samadlouie, H.; Hamidi-Esfahani, Z.; Soltani-Najafabadi, S.M.A.M.; Sahari, M.A.; Abbasi, S. Statistical approach to optimization of fermentative production of oil and arachidonic acid from Mortierella alpina CBS 754.68. Afr. J. Microbiol. Res. 2012, 6, 1559-1567.

3. Birch, E.E. Visual Acuity and the Essentiality of Docosahexaenoic Acid and Arachidonic Acid in the Diet of Term Infants. Pediatr. Res. 1998, 44, 201-209. [CrossRef]

4. Nisha, A.; Venkateswaran, G. Effect of Culture Variables on Mycelial Arachidonic acid Production by Mortierella alpina. Food Bioproc. Technol. 2011, 4, 232-240.

5. Wu, W.J.; Zhang, A.H.; Peng, C.; Ren, L.J; Song, P.; Yu, Y.D.; Huang, H.; Ji, X.J. An efficient multi-stage fermentation strategy for the production of microbial oil rich in arachidonic acid in Mortierella alpina. Bioresour. Bioprocess. 2017, 4, 8. [CrossRef]

6. Sakuradani, E.; Shimizu, S. Single cell oil production by Mortierella alpina. J. Biotechnol. 2009, 144, 31-36.

7. Wang, L.; Chen, W.; Feng, Y.; Ren, Y.; Gu, Z.; Chen, H.; Wang, H.; Thomas, M.J.; Zhang, B.; Berquin, I.M.; et al. Genome characterization of the oleaginous fungus Mortierella alpina. PLoS ONE 2011, 6, e28319. [CrossRef]

8. Etienne, K.A.; Chibucos, M.C.; Su, Q.; Orvis, J.; Daugherty, S.; Ott, S.; Sengamalay, N.A.; Fraser, C.M.; Lockhart, S.R.; Bruno, V.M. Draft genome sequence of Mortierella alpina isolate CDC-B6842. Genome Announc. 2014, 2, 2007-2008. [CrossRef] 
9. Chen, H.; Hao, G.; Wang, L.; Wang, H.; Gu, Z.; Liu, L.; Zhang, H.; Chen, W.; Chen, Y.Q. Identification of a critical determinant that enables efficient fatty acid synthesis in oleaginous fungi. Sci. Rep. 2015, 5, 11247. [CrossRef]

10. Yu, Y.; Li, T.; Wu, N.; Ren, L.; Jiang, L.; Ji, X.; Huang, H. Mechanism of Arachidonic Acid Accumulation during Aging in Mortierella alpina: A Large-Scale Label-Free Comparative Proteomics Study. J. Agric. Food Chem. 2016, 64, 9124-9134.

11. Lu, H.; Chen, H.; Tang, X.; Yang, Q.; Zhang, H.; Chen, Y.Q.; Chen, W. Time-resolved multi-omics analysis reveals the role of nutrient stress-induced resource reallocation for TAG accumulation in oleaginous fungus Mortierella alpina. Biotechnol. Biofuels 2020, 13, 116. [CrossRef]

12. Okuda, T.; Ando, A.; Negoro, H.; Kikukawa, H.; Sakamoto, T.; Sakuradani, E.; Shimizu, S.; Ogawa, J. Omega-3 eicosatetraenoic acid production by molecular breeding of the mutant strain S14 derived from Mortierella alpina 1S-4. J. Biosci. Bioeng. 2015, 120, 299-304. [CrossRef]

13. Vadivelan, G.; Venkateswaran, G. Production and enhancement of Omega-3 fatty acid from Mortierella alpina CFR-GV15: Its food and therapeutic application. Biomed Res. Int. 2014, 9, 657414.

14. Okuda, T.; Ando, A.; Negoro, H.; Muratsubaki, T.; Kikukawa, H.; Sakamoto, T.; Sakuradani, E.; Shimizu, S.; Ogawa, J. Eicosapentaenoic acid (EPA) production by an oleaginous fungus Mortierella alpina expressing heterologous the $\Delta$-17-desaturase gene under ordinary temperature. Eur. J. Lipid Sci. Technol. 2015, 117, 1919-1927. [CrossRef]

15. Ryckebosch, E.; Bruneel, C.; Muylaert, K.; Foubert, I. Microalgae as an alternative source of omega-3 long chain polyunsaturated fatty acids. Lipid Technol. 2012, 24, 128-130. [CrossRef]

16. Winwood, R.J. Recent developments in the commercial production of DHA and EPA rich oils from micro-algae. OCL Oilseeds Fats Crops Lipids 2013, 20, D604. [CrossRef]

17. Liang, Y.; Zhao, X.; Strait, M.; Wen, Z. Use of dry-milling derived thin stillage for producing eicosapentaenoic acid (EPA) by the fungus Pythium irregulare. Bioresour. Technol. 2012, 111, 404-409. [CrossRef]

18. Dedyukhina, E.G.; Chistyakova, T.I.; Mironov, A.A.; Kamzolova, S.V.; Minkevich, I.G.; Vainshtein, M.B. The effect of pH, aeration, and temperature on arachidonic acid synthesis by Mortierella alpina. Appl. Biochem. Microbiol. 2015, 51, 242-248. [CrossRef]

19. Li, X.; Lin, Y.; Chang, M.; Jin, Q.; Wang, X. Efficient production of arachidonic acid by Mortierella alpina through integrating fed-batch culture with a two-stage $\mathrm{pH}$ control strategy. Bioresour. Technol. 2015, 181, 275-282. [CrossRef]

20. Qin, X.Z.; Yang, X.P.; Chen, J.; Feng, L. Optimization of incubated mycelium of Mortierella alpina based on response surface methodology. Xinjiang Agric. Sci. 2014, 51, 89-97.

21. Huang, M.; Chen, H.; Tang, X.; Lu, H.; Chen, W. Two-stage ph control combined with oxygen-enriched air strategies for the highly efficient production of EPA by Mortierella alpina ccfm698 with fed-batch fermentation. Bioprocess Biosyst. Eng. 2020, 43 , 1725-1733. [CrossRef]

22. Nie, Z.K.; Ji, X.J.; Shang, J.S.; Zhang, A.H.; Ren, L.J.; Huang, H. Arachidonic acid-rich oil production by Mortierella alpina with different gas distributors. Bioprocess Biosyst. Eng. 2014, 37, 1127-1132. [CrossRef]

23. Ling, X.; Zeng, S.; Chen, C.; Liu, X.; Lu, Y. Enhanced arachidonic acid production using a bioreactor culture of Mortierella alpina with a combined organic nitrogen source. Bioresour. Bioprocess. 2016, 3, 43. [CrossRef]

24. Du, H.; Liao, X.; Gao, Z.; Li, Y.; Lei, Y.; Chen, W.; Chen, L.; Fan, X.; Zhang, K.; Chen, S.; et al. Effects of methanol on carotenoids as well as biomass and fatty acid biosynthesis in Schizochytrium limacinum B4D1. Appl. Environ. Microbiol. 2019, 85, 19. [CrossRef]

25. Pertea, G.; Huang, X.; Liang, F.; Antonescu, V.; Sultana, R.; Karamycheva, S.; Lee, Y.; White, J.; Cheung, F.; Parvizi, B.; et al. TIGR Gene Indices clustering tools (TGICL): A software system for fast clustering of large EST datasets. Bioinformatics 2003, 19, 651-652. [CrossRef]

26. Götz, S.; García-Gómez, J.M.; Terol, J.; Williams, T.D.; Nagaraj, S.H.; Nueda, M.J.; Robles, M.; Talón, M.; Dopazo, J.; Conesa, A. High-throughput functional annotation and data mining with the Blast2GO suite. Nucleic Acids Res. 2008, 36, 3420-3435. [CrossRef]

27. Tatusov, R.L.; Fedorova, N.D.; Jackson, J.D.; Jacobs, A.R.; Kiryutin, B.; Koonin, E.V.; Krylov, D.M.; Mazumder, R.; Mekhedov, S.L.; Nikolskaya, A.N.; et al. The COG database: An updated version includes eukaryotes. BMC Bioinform. 2003, 4, 41. [CrossRef]

28. Kanehisa, M.; Goto, S. KEGG: Kyoto encyclopedia of genes and genomes. Nucleic Acids Res. 2000, 28, 27-30. [CrossRef]

29. Dewey, C.N.; Li, B. Rsem: Accurate transcript quantification from RNA-seq data with or without a reference genome. BMC Bioinform. 2011, 12, 323.

30. Robinson, M.D.; Smyth, G.K. Moderated statistical tests for assessing differences in tag abundance. Bioinformatics 2007, 23, 2881-2887. [CrossRef]

31. Bellou, S.; Triantaphyllidou, I.E.; Aggeli, D.; Elazzazy, A.M.; Baeshen, M.N.; Aggelis, G. Microbial oils as food additives: Recent approaches for improving microbial oil production and its polyunsaturated fatty acid content. Curr. Opin. Biotechnol. 2016, 37, 24-35. [CrossRef]

32. Kothri, M.; Mavrommati, M.; Elazzazy, A.M.; Baeshen, M.N.; Moussa, T.A.A.; Aggelis, G. Microbial sources of polyunsaturated fatty acids (PUFAs) and the prospect of organic residues and wastes as growth media for PUFA-producing microorganisms. FEMS Microbiol. Lett. 2020, 367, fnaa028. [CrossRef]

33. Wongwathanarat, P.; Michaelson, L.V.; Carter, A.T.; Lazarus, C.M.; Griffiths, G.; Stobart, A.K.; Archer, D.B.; MacKenzie, D.A. Two fatty acid Delta 9-desaturase genes, ole1 and ole2, from Mortierella alpina complement the yeast ole1 mutation. Microbiology 1999, 145, 2939-2946. [CrossRef] 
34. Hao, G.; Chen, H.; Yang, B.; Du, K.; Wang, H.; Gu, Z.; Zhang, H.; Chen, W.; Chen, Y.Q. Substrate specificity of Mortierella alpina $\Delta$-9-III fatty acid desaturase and its value for the production of omega-9 MUFA. Eur. J. Lipid Sci. Technol. 2016, 118, 753-760. [CrossRef]

35. Huang, Y.S.; Chaudhary, S.; Thurmond, J.M.; Bobik, E.G.; Yuan, L.; Chan, G.M.; Kirchner, S.J.; Mukerji, P.; Knutzon, D.S. Cloning of Delta 12-and Delta 6- desaturases from Mortierella alpina and recombinant production of gamma-linolenic acid in Saccharomyces cerevisiae. Lipids 1999, 34, 649-659. [CrossRef]

36. Wang, C.; Qing, X.; Yu, M.; Sun, Q.; Liu, F.; Qi, B.; Li, X. Production of eicosapentaenoic acid (EPA, 20:5n-3) in transgenic peanut (Arachis hypogaea L.) through the alternative Delta 8-desaturase pathway. Mol. Biol. Rep. 2019, 46, 333-342. [CrossRef]

37. Sakuradani, E.; Abe, T.; Iguchi, K.; Shimizu, S. A novel fungal omega 3-desaturase with wide substrate specificity from arachidonic acid-producing Mortierella alpina 1S-4. Appl. Microbiol. Biotechnol. 2005, 66, 648-654. [CrossRef]

38. Tv, A.; Ct, A.; Tp, B.; Ts, C.; Pj, D.; Tw, D. Systematic genome analysis of a novel arachidonic acid-producing strain uncovered unique metabolic traits in the production of acetyl-CoA-derived products in mortierellale fungi. Gene 2020, 741, 144550.

39. Chang, L.; Chen, H.; Tang, X.; Zhao, J.; Zhang, H.; Chen, Y.Q.; Chen, W. Advances in improving the biotechnological application of oleaginous fungus Mortierella alpina. Appl. Microbiol. Biotechnol. 2021, 105, 6275-6289. [CrossRef]

40. Houten, S.M.; Wanders, R.J.A. A general introduction to the biochemistry of mitochondrial fatty acid $\beta$-oxidation. J. Inherit. Metab. Dis. 2010, 33, 469-477. [CrossRef]

41. Zhang, Y.; Adams, I.P.; Ratledge, C. Malic enzyme: The controlling activity for lipid production? Overexpression of malic enzyme in Mucor circinelloides leads to a 2.5-fold increase in lipid accumulation. Microbiology 2007, 153, 2013-2025. [CrossRef] 